\title{
Szegő orthogonal polynomials with respect to an analytic weight: canonical representation and strong asymptotics.
}

\author{
A. Martínez-Finkelshtein* \\ K. T.-R. McLaughlin \\ Universidad de Almería, Spain \\ University of Arizona, USA \\ E. B. Saff \\ Vanderbilt University, USA
}

February 8, 2008

\begin{abstract}
We provide a representation in terms of certain canonical functions for a sequence of polynomials orthogonal with respect to a weight that is strictly positive and analytic on the unit circle. These formulas yield a complete asymptotic expansion for these polynomials, valid uniformly in the whole complex plane. As a consequence, we obtain some results about the distribution of zeros of these polynomials. The main technique is the steepest descent analysis of Deift and Zhou, based on the matrix Riemann-Hilbert characterization proposed by Fokas, Its and Kitaev.
\end{abstract}

\section{Introduction and background}

For $r>0$, let $\mathbb{D}_{r}$ be the open disc in $\mathbb{C}$ with center at $z=0$ and radius $r,\{z \in \mathbb{C}:|z|<r\}$, and let $\mathbb{T}_{r}$ be its boundary $\{z \in \mathbb{C}:|z|=r\}$. An integrable non-negative function $w$ defined on $\mathbb{T}_{1}$ is called a weight if

$$
\int_{\mathbb{T}_{1}} w(z)|d z|>0
$$

For each weight $w$ there exists a unique sequence of polynomials $\varphi_{n}$ (called Szegö polynomials), orthonormal with respect to $w$, satisfying $\varphi_{n}(z)=\kappa_{n} z^{n}+$ lower degree terms, $\kappa_{n}>0$, and

$$
\oint_{\mathbb{T}_{1}} \varphi_{n}(z) \overline{\varphi_{m}(z)} w(z)|d z|=\delta_{m n} .
$$

\footnotetext{
${ }^{*}$ Corresponding author. Email: andrei@ual.es
} 
We denote by $\Phi_{n}(z) \stackrel{\text { def }}{=} \varphi_{n}(z) / \kappa_{n}$ the corresponding monic orthogonal polynomials. It is well known that they satisfy the Szegö recurrence

$$
\Phi_{n+1}(z)=z \Phi_{n}(z)-\overline{\alpha_{n}} \Phi_{n}^{*}(z), \quad \Phi_{0}(z) \equiv 1,
$$

where we use the standard notation $\Phi_{n}^{*}(z) \stackrel{\text { def }}{=} z^{n} \overline{\Phi_{n}(1 / \bar{z})}$. The parameters $\alpha_{n}=-\overline{\Phi_{n+1}(0)}$ are called Verblunsky coefficients (also reflection coefficients or Schur parameters) and satisfy $\alpha_{n} \in \mathbb{D}_{1}$ for $n=0,1,2, \ldots$ (see Sim04a for details).

The asymptotics of the sequence $\left\{\varphi_{n}(z)\right\}$ when $n \rightarrow \infty$ is well known for $|z|>1$ (the so called outer or exterior asymptotics) under rather general assumptions on the weight. However, the behavior of these polynomials in $\mathbb{D}_{1}$ is, in general, much more complicated and less studied. First, it is known that all the zeros of each $\Phi_{n}$ lie in $\mathbb{D}_{1}$ (see e.g. [GS84, p. 50-55], Sze75. Ch. XII, theorem 12.1.1], Ger61] or Sim04a]). Moreover, they can be everywhere dense in the unit disc (the so-called Turan's conjecture), as shown in AV88. A recent result of Simon and Totik ST04 proves even the existence of a "universal" measure of orthogonality on $\mathbb{T}_{1}$ for which the zero distributions of the corresponding $\left\{\Phi_{n}(z)\right\}$ approximate (in a weak sense) all the possible measures on the closed disc $\overline{\mathbb{D}}_{1}$.

However, under stronger assumptions on the weight $w$ we can say much more. In what follows we will consider only the strictly positive weights $w$ on the unit circle that can be extended to an open set containing $\mathbb{T}_{1}$ as a nowhere vanishing holomorphic function. We will say briefly that such $w$ is a strictly positive analytic weight on $\mathbb{T}_{1}$. All the forthcoming statements are valid under this assumption. In order to shed light on this definition we point out that $w$ is a non-vanishing holomorphic function in an annulus $r<|z|<1 / r$ (with $0<r<1$ ) and strictly positive on the unit circle $\mathbb{T}_{1}$, if and only if, for any nonzero $\alpha \in \mathbb{R}$ the following representation is valid on $\mathbb{T}_{1}$ :

$$
w(z)=|W(z)|^{\alpha}, \quad z \in \mathbb{T}_{1},
$$

where $W$ is holomorphic and nowhere vanishing in the annulus. In particular, for $\alpha=2$, the following representation is useful:

$$
w(z)=W(z) \overline{W(z)}=W(z) \overline{W(1 / \bar{z})}, \quad z \in \mathbb{T}_{1} .
$$

Obviously, here $W$ is not uniquely defined. Normalized representations of this form arise from the so-called Szegö function of $w$. Indeed, since $w$ is strictly positive on $\mathbb{T}_{1}$, its index (winding number about the origin) is 0, and we may define the Szegö function (see e.g. Sze75 Ch. X, $\S 10.2])$ :

$$
D(z) \stackrel{\text { def }}{=} \exp \left(\frac{1}{4 \pi} \int_{0}^{2 \pi} \log w\left(e^{i \theta}\right) \frac{e^{i \theta}+z}{e^{i \theta}-z} d \theta\right) .
$$

This function is piecewise analytic and non-vanishing, defined for $|z| \neq 1$, and we will denote by $D_{\mathrm{i}}$ and $D_{\mathrm{e}}$ its values for $|z|<1$ and $|z|>1$, respectively. It is easy to verify that

$$
\overline{D_{\mathrm{i}}\left(\frac{1}{\bar{z}}\right)}=\frac{1}{D_{\mathrm{e}}(z)}, \quad|z|>1 \text {. }
$$


Furthermore, if $w$ satisfies the assumptions above, both $D_{\mathrm{i}}$ and $D_{\mathrm{e}}$ admit a holomorphic extension across $\mathbb{T}_{1}$, and maintaining the same notation for these analytic continuations we have

$$
\frac{D_{\mathrm{i}}(z)}{D_{\mathrm{e}}(z)}=w(z)
$$

This formula gives an analytic extension of $w$ from $\mathbb{T}_{1}$ to an open domain containing the unit circle and, up to a normalization constant, provides the "standard" representation (3). Observe that by (5), $w(z)=\left|D_{\mathrm{e}}(z)\right|^{-2}$ for $z \in \mathbb{T}_{1}$.

Let us define

$$
\rho \stackrel{\text { def }}{=} \inf \left\{0<r<1: D_{\mathrm{e}}(z) \text { is holomorphic in }|z|>r\right\} .
$$

The class of weights that we consider in this article is characterized by the fact that $\rho<1$. Due to the symmetry (5), $1 / \rho$ is the radius of convergence of the Taylor series for $1 / D_{\mathrm{i}}$ about $z=0$ (hence, the constant $1 / \rho$ coincides with $R_{D^{-1}}$ in the notation of Sim04a, $\left.\S 7.1\right]$ ). Taking into account (6), we may equivalently define $\rho$ as

$$
\rho=\inf \{0<r<1: 1 / w(z) \text { is holomorphic in } r<|z|<1 / r\} .
$$

Again by (5), both circles $\mathbb{T}_{\rho}$ and $\mathbb{T}_{1 / \rho}$ contain singularities of $1 / w$.

Important results concerning the class of strictly positive analytic weights on $\mathbb{T}_{1}$ were obtained in [NT89]. It was shown there (see also [Sim04a, §7.1]) that

$$
\rho=\varlimsup_{n \rightarrow \infty}\left|\alpha_{n}\right|^{1 / n} .
$$

In particular, strictly positive analytic weights on $\mathbb{T}_{1}$ are characterized by an exponential decay of the corresponding Verblunsky coefficients. Furthermore, the Szegő asymptotic formula

$$
\lim _{n \rightarrow \infty} \varphi_{n}^{*}(z)=\frac{1}{D_{\mathrm{i}}(z)}, \quad z \in \mathbb{D}_{1},
$$

can be continued analytically through the unit circle $\mathbb{T}_{1}$ and is valid locally uniformly in $\mathbb{D}_{1 / \rho}$. It shows that the number of zeros of $\left\{\varphi_{n}\right\}$ outside of $\overline{\mathbb{D}}_{\rho}$ remains uniformly bounded and these zeros are attracted by the zeros of $D_{\mathrm{e}}$ in $\rho<|z|<1$ (Nevai-Totik points in the terminology of B. Simon Sim04b]). Mhaskar and Saff [MS90, using potential theory arguments, gave a relevant complement to these results about zeros showing that for any subsequence $\left\{n_{k}\right\} \subset \mathbb{N}$ satisfying

$$
\rho=\lim \left|\alpha_{n_{k}}\right|^{1 / n_{k}},
$$

the zeros of $\left\{\varphi_{n_{k}+1}\right\}$ distribute asymptotically uniformly in the weak-star sense on $\mathbb{T}_{\rho}$ (see also Pak87].).

Further results on the asymptotics or zero behavior inside $\mathbb{T}_{\rho}$ have been obtained so far only for some particular subclasses of strictly positive analytic weights on $\mathbb{T}_{1}$. Besides the 
classical explicit formula for the Bernstein-Szegö polynomials (when $w(z)=|1 / q(z)|^{2}, q$ a fixed polynomial with zeros in $\mathbb{C} \backslash \mathbb{T}_{1}$ ), probably the first important result in this direction was obtained by Szabados [Sza79], who considered the polynomial case $w(z)=|p(z)|^{2}$ (where again $p$ is a fixed polynomial with zeros in $\mathbb{C} \backslash \mathbb{T}_{1}$ ). Szabados gave a formula for the orthogonal polynomials with respect to $|p(z)|^{2}$ which, although containing unknown parameters, is sufficient for the asymptotic analysis inside $\mathbb{T}_{\rho}$.

By extending Darboux's formula to Szegő orthogonal polynomials, Ismail and Ruedemann IR92 (see also GM91) obtain matrix expressions for the $\varphi_{n}$ 's for the case of rational weights $w(z)=|p(z) / q(z)|^{2}$, where the size of the matrix depends on the degrees of $p$ and $q$. They applied their result to the asymptotic analysis in the case when $\mathbb{T}_{\rho}$ contains a single zero of $w$. The same rational case is also the object of study in a less known paper [Pak87], where the author considers the weak-star zero distribution for the $\varphi_{n}$ 's.

Another class of weights for which $\rho<1$ corresponds to the Rogers-Szegö polynomials, studied for instance, in MGH90, where even the interlacing properties of their zeros have been established (see also [LS87] and [Sim04a, Example 1.6.5]). The reader interested in many more explicit examples of orthogonal polynomials on $\mathbb{T}_{1}$ is referred to Sim04a, Ch. I and VIII].

The main goal of this paper is to provide a complete asymptotic expansion for the orthogonal polynomials, valid uniformly in the whole complex plane, under the assumption that $w$ is a strictly positive analytic weight on $\mathbb{T}_{1}$. As a consequence, we obtain results about the distribution of zeros of these orthogonal polynomials, under a variety of different assumptions about the weight function. These results recover, in particular cases, some of the results mentioned above. The main technique is the steepest descent analysis of Deift and Zhou (see e.g. Dei99a]), based on the matrix Riemann-Hilbert characterization proposed by Fokas, Its and Kitaev FIK92. This approach more generally allows one to handle analytic complex valued weights $w$ that are nonvanishing on $\mathbb{T}_{1}$ provided the corresponding orthonormal polynomials $\varphi_{n}(z)$ exist with $\kappa_{n} \neq 0$ for each $n$. Furthermore, the asymptotic analysis is equally possible (and technically not much more involved) for a sequence of varying weights (when $w$ depends on the degree $n$ of the polynomial). This was carried out previously in BDJ99 for a specific weight relevant to the asymptotics of the distribution of the length of the longest increasing sequences in random permutations. A basic discussion of orthogonal polynomials on the unit circle, in the context of an example of integrable operators, and their connection with the Riemann-Hilbert problems is contained in Dei99b.

Until recently the analyticity of $w$ has been a necessary condition for the applicability of the Riemann-Hilbert asymptotic analysis. However, McLaughlin and Miller [MM04 have extended the approach via the solution of a d-bar problem. Their method allows one to handle piecewise smooth and strictly positive weights $w$ on $\mathbb{T}_{1}$; for this generality they pay the price of less detailed asymptotics. Also their results are qualitatively different: in the settings of MM04 essentially all the zeros approach $\mathbb{T}_{1}$ with a polynomial rate depending on the smoothness of $w$, and those that might stay inside are artifacts of the jumps of the 
weight.

Our asymptotic formulas (Theorem 1) are stated in terms of a sequence of iterated Cauchy transforms of the function

$$
\mathcal{F}(z) \stackrel{\text { def }}{=} D_{\mathrm{i}}(z) D_{\mathrm{e}}(z),
$$

where $D_{\mathrm{i}}$ and $D_{\mathrm{e}}$ are respectively the inner and the outer Szegö functions defined by (4). By (17), $\mathcal{F}$ is holomorphic in $\rho<|z|<1$. Furthermore, by (51),

$$
\overline{\mathcal{F}\left(\frac{1}{\bar{z}}\right)}=\frac{1}{\mathcal{F}(z)}, \quad \text { for }|z| \neq 1, \quad|\mathcal{F}(z)|=1 \text { on } \mathbb{T}_{1}
$$

and $1 / \mathcal{F}$ is holomorphic in $1<|z|<1 / \rho$.

The function $1 / \mathcal{F}$ appears as a scattering matrix (or function) in [GC79, Formula (V.5)]. Geronimo and Case also give an asymptotic expression for $\varphi_{n}$ on $\mathbb{T}_{1}$, where the Cauchy transform of the scattering functions appears implicitly (cf. formulas (III.7) and (V.8) in GC79]). One of our goals here is to make precise the relevant role of the sequences of iterated Cauchy transforms related to $\mathcal{F}$ in the asymptotics of the orthogonal polynomials.

The structure of the paper is as follows. In the next section we state the main results giving a representation of the polynomials $\Phi_{n}$, the leading coefficients $\kappa_{n}$ and the Verblunsky coefficients $\alpha_{n}$ in terms of the series of some canonical functions. These formulas have an asymptotic nature that we exploit in Section [3, where we present a number of results concerning the asymptotic behavior of the Verblunsky coefficients, leading coefficients, and the "outer" asymptotic behavior of the orthogonal polynomials. We also present in Section 3 general results describing the asymptotic behavior of the zeros, for a rather wide class of analytic weights. The detailed assumption is that the exterior function $D_{\mathrm{e}}$ can be extended to the exterior of a domain $\left\{|z|>\rho^{\prime}\right\}$ with finitely many poles. The proofs of these results are deferred to Section 4. Finally, in Section 5] we discuss some particular cases, including the case of a weight for which $D_{e}$ possesses an isolated essential singularity within the unit disk. The examples in Section [5] are all illustrated also with numerical experiments. We should point out that the zeros of $\Phi_{n}$ 's have been computed as the eigenvalues of the truncated matrix corresponding to the CMV representation of the multiplication operator (see CMV03 or [Sim04a, Ch. IV] for details), using a symbolic algebra software with extended precision. 


\section{Canonical representation of the orthogonal polynomials}

Occasionally it is more natural to write our formulas in terms of a modified Szegő function. Since $w$ is of index 0 , we may define the calligraphic functions

$$
\begin{array}{ll}
\mathcal{D}_{\mathrm{i}}(z) \stackrel{\text { def }}{=} \exp \left(\frac{1}{2 \pi i} \oint_{\mathbb{T}_{1}} \frac{\log w(t) d t}{t-z}\right), & \text { if }|z|<1, \\
\mathcal{D}_{\mathrm{e}}(z) \stackrel{\text { def }}{=} \exp \left(-\frac{1}{2 \pi i} \oint_{\mathbb{T}_{1}} \frac{\log w(t) d t}{t-z}\right), & \text { if }|z|>1 .
\end{array}
$$

Both $\mathcal{D}_{\mathrm{i}}$ and $\mathcal{D}_{\mathrm{e}}$ are holomorphic and non-vanishing in the respective regions where they are defined and satisfy

$$
\overline{\tau \mathcal{D}_{\mathrm{i}}\left(\frac{1}{\bar{z}}\right)}=\frac{\mathcal{D}_{\mathrm{e}}(z)}{\tau}, \quad|z|>1
$$

where $\tau$ is the Szegő constant (mean value) given by

$$
\tau \stackrel{\text { def }}{=} \frac{1}{D_{\mathrm{i}}(0)}=\exp \left(-\frac{1}{4 \pi} \int_{0}^{2 \pi} \log w\left(e^{i \theta}\right) d \theta\right)=\exp \left(-\frac{1}{4 \pi i} \int_{\mathbb{T}_{1}} \log w(t) \frac{d t}{t}\right)>0 .
$$

Furthermore (compare (6) ),

$$
\mathcal{D}_{\mathrm{i}}(z) \mathcal{D}_{\mathrm{e}}(z)=w(z)
$$

This equation plus the normalization $\mathcal{D}_{\mathrm{e}}(\infty)=1$ determines uniquely the nowhere vanishing functions (13)-(14). Finally, we have the following straightforward identities for $|z| \neq 1$ :

$$
\begin{array}{llrl}
D_{\mathrm{i}}(z) & =\tau \mathcal{D}_{\mathrm{i}}(z), & & \text { if }|z|<1, \\
D_{\mathrm{e}}(z) & =\tau \mathcal{D}_{\mathrm{e}}(z)^{-1}, & & \text { if }|z|>1 .
\end{array}
$$

In what follows, all the circles $\mathbb{T}_{\alpha}, \alpha>0$, are oriented counterclockwise. For a fixed value of $\alpha$ (which will be clear from the context), we set

$$
f_{+}(z) \stackrel{\text { def }}{=} \lim _{\substack{u \rightarrow z \\|u|<\alpha}} f(t), \quad f_{-}(z) \stackrel{\text { def }}{=} \lim _{\substack{u \rightarrow z \\|u|>\alpha}} f(t),
$$

for any analytic function $f$ for which these limits exist. We will also talk about the "+" and the "-" side of $\mathbb{T}_{\alpha}$ referring to its inner and outer boundary points, respectively.

Choose an arbitrary $r, \rho<r<1$, that we fix for what follows. We introduce the following operators acting on the space of holomorphic functions in $\mathbb{C} \backslash\left(\mathbb{T}_{r} \cup \mathbb{T}_{1 / r}\right)$ with continuous boundary values:

$$
\begin{aligned}
& \mathcal{M}_{n}^{\mathrm{i}}(f)(z) \stackrel{\text { def }}{=}-\frac{1}{2 \pi i} \oint_{\mathbb{T}_{r}} f_{-}(t) \frac{t^{n} \mathcal{D}_{\mathrm{i}}^{2}(t)}{w(t)(t-z)} d t=-\frac{1}{2 \pi i \tau^{2}} \oint_{\mathbb{T}_{r}} f_{-}(t) \frac{\mathcal{F}(t) t^{n}}{t-z} d t \\
& \mathcal{M}_{n}^{\mathrm{e}}(f)(z) \stackrel{\text { def }}{=} \frac{1}{2 \pi i} \oint_{\mathbb{T}_{1 / r}} f_{-}(t) \frac{\mathcal{D}_{\mathrm{e}}^{2}(t)}{t^{n} w(t)(t-z)} d t=\frac{\tau^{2}}{2 \pi i} \oint_{\mathbb{T}_{1 / r}} f_{-}(t) \frac{1}{\mathcal{F}(t) t^{n}(t-z)} d t
\end{aligned}
$$


where $f_{-}$denotes the exterior boundary values of the function $f$ on the respective circles.

Let us denote by

$$
\Lambda \stackrel{\text { def }}{=}\|\mathcal{F}\|_{\mathbb{T}_{r}}=\max _{z \in \mathbb{T}_{r}}|\mathcal{F}(z)|
$$

taking into account (12), also

$$
\Lambda=\left\|\frac{1}{\mathcal{F}}\right\|_{\mathbb{T}_{1 / r}}=\max _{z \in \mathbb{T}_{1 / r}} \frac{1}{|\mathcal{F}(z)|} .
$$

Then straightforward bounds show that

$$
\begin{aligned}
& \left|\mathcal{M}_{n}^{\mathrm{i}}(f)(z)\right| \leq \frac{\Lambda r}{\tau^{2}} r^{n} \frac{\left\|f_{-}\right\|_{\mathbb{T}_{r}}}{|| z|-r|}, \quad z \notin \mathbb{T}_{r}, \\
& \left|\mathcal{M}_{n}^{\mathrm{e}}(f)(z)\right| \leq \frac{\Lambda \tau^{2}}{r} r^{n} \frac{\left\|f_{-}\right\|_{\mathbb{T}_{1 / r}}}{|| z|-1 / r|}, \quad z \notin \mathbb{T}_{1 / r} .
\end{aligned}
$$

Remark 1 It is obvious that for $|z|<\rho$ in (18) and for $|z|>1 / \rho$ in (19) we may take $\mathbb{T}_{1}$ as the contour of integration in these formulas. However, our choice makes inequalities (21) evident.

Using these operators we define the following sequence of functions:

$$
f_{n}^{(0)} \stackrel{\text { def }}{=} 1, \quad f_{n}^{(1)} \stackrel{\text { def }}{=} \mathcal{M}_{n}^{\mathrm{i}}(1), \quad f_{n}^{(2)} \stackrel{\text { def }}{=} \mathcal{M}_{n}^{\mathrm{e}}\left(f_{n}^{(1)}\right),
$$

and

$$
f_{n}^{(2 k+1)} \stackrel{\text { def }}{=} \mathcal{M}_{n}^{\mathrm{i}}\left(f_{n}^{(2 k)}\right), \quad f_{n}^{(2 k+2)} \stackrel{\text { def }}{=} \mathcal{M}_{n}^{\mathrm{e}}\left(f_{n}^{(2 k+1)}\right), \quad k \in \mathbb{N} .
$$

Observe that each of these formulas defines in fact two analytic functions, one inside and one outside of the circle where the corresponding integral is taken. In order to avoid cumbersome notation we will not use the subindices $i$ and e in this case, but the reader should bear in mind this fact.

By (21),

$$
\begin{aligned}
|z| \neq r & \Rightarrow\left|f_{n}^{(1)}\right|=\left|\mathcal{M}_{n}^{\mathrm{i}}(1)\right| \leq \frac{\Lambda r}{\tau^{2}} \frac{r^{n}}{|| z|-r|}, \\
|z| \neq 1 / r & \Rightarrow\left|f_{n}^{(2)}\right|=\left|\mathcal{M}_{n}^{\mathrm{e}}\left(f_{n}^{(1)}\right)\right| \leq \frac{\Lambda \tau^{2}}{r} \frac{r^{n}}{|| z|-1 / r|}\left\|f_{n}^{(1)}\right\|_{\mathbb{T}_{1 / r}} \leq \frac{\Lambda^{2} r^{2 n}}{\frac{1}{r}-r} \frac{1}{\| z|-1 / r|} \\
|z| \neq r & \Rightarrow\left|f_{n}^{(3)}\right|=\left|\mathcal{M}_{n}^{\mathrm{i}}\left(f_{n}^{(2)}\right)\right| \leq \frac{\Lambda r}{\tau^{2}} \frac{r^{n}}{|| z|-r|}\left\|f_{n}^{(2)}\right\|_{\mathbb{T}_{r}} \leq \frac{r}{\tau^{2}} \frac{\Lambda^{3} r^{3 n}}{\left(\frac{1}{r}-r\right)^{2}} \frac{1}{\| z|-r|},
\end{aligned}
$$


and in general, for $k \in \mathbb{N}$,

$$
\left|f_{n}^{(k)}(z)\right| \leq \begin{cases}\frac{r}{\tau^{2}} \frac{\Lambda^{k} r^{k n}}{\left(\frac{1}{r}-r\right)^{k-1}} \frac{1}{|| z|-r|}, & \text { if } k \text { is odd, and }|z| \neq r \\ \frac{\Lambda^{k} r^{k n}}{\left(\frac{1}{r}-r\right)^{k-1}} \frac{1}{|| z|-1 / r|}, & \text { if } k \text { is even, and }|z| \neq 1 / r\end{cases}
$$

Remark 2 There are a number of equivalent definitions for the functions $f_{n}^{(1)}$. For instance,

$$
-f_{n}^{(1)}(z)=\frac{1}{2 \pi i} \oint_{\mathbb{T}_{r}} \frac{t^{n} \mathcal{D}_{\mathrm{i}}^{2}(t)}{w(t)(t-z)} d t=\frac{1}{2 \pi i \tau^{2}} \oint_{\mathbb{T}_{r}} \frac{t^{n} \mathcal{F}(t)}{t-z} d t=\frac{1}{2 \pi i \tau^{2}} \oint_{\mathbb{T}_{1}} \frac{t^{n} \mathcal{F}(t)}{t-z} d t .
$$

We can rewrite the integrand directly in terms of the weight $w$ recalling that on $\mathbb{T}_{1}$,

$$
\frac{\mathcal{F}(t)}{\tau^{2}}=\exp \left(\frac{1}{\pi i} f_{\mathbb{T}_{1}} \frac{\log w(s) d s}{s-t}\right),
$$

where the integral is understood as its principal value. If

$$
\mathcal{F}(z)=\sum_{k=-\infty}^{+\infty} c_{k} z^{k}
$$

is the Laurent expansion of $\mathcal{F}$ in the annulus $\rho<|z|<1 / \rho$ and $\mathcal{P}_{+}$is the Riesz projection onto $H^{2}\left(\mathbb{D}_{1}\right)$, then for $z \in \mathbb{D}_{r}$,

$$
f_{n}^{(1)}(z)=-\frac{1}{\tau^{2}} \mathcal{P}_{+}\left(z^{n} \mathcal{F}(z)\right)=-\frac{z^{n}}{\tau^{2}} \times\left(\text { Laurent series of } \mathcal{F} \text { truncated at } z^{-n}\right)
$$

which establishes a connection of the $f_{n}^{(k)}$ 's with Hankel operators. It also shows that for $\rho<|z|<r$,

$$
\lim _{n \rightarrow \infty} \frac{f_{n}^{(1)}(z)}{z^{n}}=-\frac{\mathcal{F}(z)}{\tau^{2}}
$$

and convergence is uniform on each compact subset of this annulus.

Finally, taking into account that

$$
f_{n}^{(1)}(0)=-\frac{1}{2 \pi i \tau^{2}} \oint_{\mathbb{T}_{1}} t^{n-1} \mathcal{F}(t) d t=-\frac{c_{-n}}{\tau^{2}},
$$

it is easy to see that sequence $\left\{f_{n}^{(1)}\right\}$ satisfies the following recurrence:

$$
f_{n+1}^{(1)}(z)=z f_{n}^{(1)}-\frac{c_{-n-1}}{\tau^{2}}, \quad z \in \mathbb{D}_{1} .
$$


Let

$$
\mathcal{E}_{n}(z) \stackrel{\text { def }}{=} \sum_{k=0}^{\infty} f_{n}^{(2 k)}(z), \quad|z| \neq 1 / r, \quad \text { and } \quad \mathcal{I}_{n}(z) \stackrel{\text { def }}{=} \sum_{k=0}^{\infty} f_{n}^{(2 k+1)}(z), \quad|z| \neq r .
$$

Bounds (24) show that the series in the right hand sides of (29) converge absolutely and locally uniformly in the respective regions of definition if

$$
\Lambda r^{n}<\frac{1}{r}-r \Longleftrightarrow n>\frac{\log \left(\frac{1 / r-r}{\Lambda}\right)}{\log r} .
$$

In this case there exists a constant $C>0$ depending on $r$ and $\Lambda$ only, such that

$$
\left|\mathcal{E}_{n}(z)\right| \leq \frac{C}{|| z|-1 / r|}, \text { for }|z| \neq 1 / r, \quad \text { and } \quad\left|\mathcal{I}_{n}(z)\right| \leq \frac{C}{|| z|-r|} \text {, for }|z| \neq r .
$$

In the sequel we use $C$ (eventually, with a subindex) to denote some irrelevant constants, different in each appearance, whose dependence or independence on the parameters will be stated explicitly.

Now we may state one of the main results of the paper:

Theorem 1 Let $w$ be a strictly positive analytic weight on the unit circle $\mathbb{T}_{1}$, the constant $\rho$ as defined in (7) (8), and constant $r$ with $\rho<r<1$ fixed. Then with the notations introduced above, for every $n \in \mathbb{N}$ satisfying (30) the following formulas hold:

$$
\Phi_{n}(z)= \begin{cases}\tau^{-1} z^{n} D_{\mathrm{e}}(z) \mathcal{E}_{n}(z), & \text { if }|z|>1 / r \\ \tau^{-1} z^{n} D_{\mathrm{e}}(z) \mathcal{E}_{n}(z)-\frac{\tau \mathcal{I}_{n}(z)}{D_{\mathrm{i}}(z)}, & \text { if } r<|z|<1 / r \\ -\frac{\tau \mathcal{I}_{n}(z)}{D_{\mathrm{i}}(z)}, & \text { if }|z|<r .\end{cases}
$$

We emphasize that in each of the regions above $\mathcal{E}_{n}$ and $\mathcal{I}_{n}$ have the meaning given in (29), and are not obtained in general by analytic continuation from one domain to another.

Remark 3 The canonical formulae expressed in (32) appear to be valid for $z$ bounded away from the circles of radius $r$ and $1 / r$. However, Theorem 1 holds true for any $r$ with $\rho<r<1$, and so the formulae, in fact, are valid up to the boundaries, by analytic continuation. Indeed, by considering $\hat{r}$ with $r<\hat{r}<1$, the last formula in (32) is seen to remain valid by analytic continuation, for $|z|<\hat{r}$. Similarly, the first formula is valid by analytic continuation for $|z|>1 / \hat{r}$. For the middle formula in (32), one considers $\tilde{r}$ with $\rho<\tilde{r}<r$, which effectively extends the region where the middle formula is valid. Therefore, the formulae contained in (32) are each in fact valid on the closure of the domains stated. 
Remark 4 The second formula in (32) expresses the polynomial $\Phi_{n}$ on $\mathbb{T}_{1}$ as a sum of "inner" and "outer" terms. An alternative two-term expression for $\Phi_{n}$ on $\mathbb{T}_{1}$ can be found in [GC79, Formula (III.7)]. In the latter formula we can identify the inner and outer Szegö functions, but the other factors are given implicitly in terms of the solutions of equations from the inverse scattering theory.

Taking into account (16), the following representation of the Verblunsky coefficients is straightforward:

Corollary 1 With $w$ as in Theorem 1, we have for each $n \in \mathbb{N}$,

$$
\overline{\alpha_{n}}=\tau^{2} \mathcal{I}_{n+1}(0)=\tau^{2} \sum_{k=0}^{\infty} f_{n+1}^{(2 k+1)}(0) .
$$

Observe that under assumptions (30) the series on the right hand side converges absolutely.

The Riemann-Hilbert asymptotic analysis that we carry out in Section 4 allows us to find also a representation for the leading coefficients of the orthonormal polynomials. For this purpose we define the "shifted" sequence with respect to (22)-(23):

$$
g_{n}^{(0)} \stackrel{\text { def }}{=} 1, \quad g_{n}^{(1)} \stackrel{\text { def }}{=} \mathcal{M}_{n}^{\mathrm{e}}(1), \quad g_{n}^{(2)} \stackrel{\text { def }}{=} \mathcal{M}_{n}^{\mathrm{i}}\left(g_{n}^{(1)}\right),
$$

and

$$
g_{n}^{(2 k+1)} \stackrel{\text { def }}{=} \mathcal{M}_{n}^{\mathrm{e}}\left(g_{n}^{(2 k)}\right), \quad g_{n}^{(2 k+2)} \stackrel{\text { def }}{=} \mathcal{M}_{n}^{\mathrm{i}}\left(g_{n}^{(2 k+1)}\right), \quad k \in \mathbb{N} .
$$

Inequalities (21) imply again that for $k \in \mathbb{N}$,

$$
\left|g_{n}^{(2 k)}(0)\right| \leq \frac{\Lambda^{2 k} r^{2 k n-1}}{\left(\frac{1}{r}-r\right)^{2 k-1}} .
$$

Theorem 2 With $w$ as in Theorem 1, and for $n$ satisfying (30), the leading coefficient $\kappa_{n}$ of $\varphi_{n}$ has the following representation:

$$
\kappa_{n}^{2}=\frac{\tau^{2}}{2 \pi} \sum_{k=0}^{\infty} g_{n}^{(2 k)}(0) .
$$




\section{Asymptotic behavior}

By (24) it is immediate that for $n$ satisfying (30) and for $N=0,1,2, \ldots$,

$$
\left|\mathcal{E}_{n}(z)-\sum_{k=0}^{N} f_{n}^{(2 k)}(z)\right| \leq \frac{C_{N}}{|| z|-1 / r|} r^{(2 N+2) n}, \quad|z| \neq 1 / r,
$$

where the constant $C_{N}$ depends only on $r, N$ and $\Lambda$, but neither on $n$ nor on $z$. Analogously,

$$
\left|\mathcal{I}_{n}(z)-\sum_{k=0}^{N} f_{n}^{(2 k+1)}(z)\right| \leq \frac{C_{N}}{|| z|-r|} r^{(2 N+3) n}, \quad|z| \neq r,
$$

where $C_{N}$ has a similar meaning as above. Here a similar observation concerning the validity of these bounds up to the boundary, made in Remark 3, applies. These bounds show that (32) allows us to obtain an asymptotic expression for $\left\{\Phi_{n}\right\}$ of an arbitrarily high order. Let us discuss the consequences of truncating $\mathcal{I}_{n}$ and $\mathcal{E}_{n}$ in (32) at their first terms.

For instance, from Corollary 1 and Theorem 2 we get the following estimates of the Verblunsky coefficients $\alpha_{n}$ and the leading coefficients $\kappa_{n}$ :

Corollary 2 Let $w$ be a strictly positive analytic weight on the unit circle $\mathbb{T}_{1}$. With the notation introduced above and for each $n \in \mathbb{N}$,

$$
\overline{\alpha_{n}}=-\frac{1}{2 \pi i} \oint_{\mathbb{T}_{r}} t^{n} \mathcal{F}(t) d t+\mathcal{O}\left(r^{3 n}\right)=-c_{-n-1}+\mathcal{O}\left(r^{3 n}\right)
$$

where $c_{-n-1}$ is the corresponding Laurent coefficient of $\mathcal{F}$ in (26). Consequently,

$$
\left|\alpha_{n}\right| \leq \Lambda r^{n+1}+\mathcal{O}\left(r^{3 n}\right) .
$$

Furthermore,

$$
\left|\kappa_{n}-\frac{\tau^{2}}{2 \pi}\right| \leq \frac{\Lambda^{2} r^{2 n}}{1-r^{2}}
$$

Another straightforward consequence of truncating $\mathcal{I}_{n}$ and $\mathcal{E}_{n}$ at their first terms is the exterior asymptotics of $\left\{\Phi_{n}\right\}$ :

Proposition 1 Let $w$ be a strictly positive analytic weight on the unit circle $\mathbb{T}_{1}$, and the constant $\rho$ as defined in (77)-(8). Then

$$
\lim _{n \rightarrow \infty} \frac{\Phi_{n}(z)}{z^{n}}=\frac{D_{\mathrm{e}}(z)}{\tau}
$$


locally uniformly for $|z|>\rho$ and convergence takes place with a geometric rate. In particular, for any compact set $K$ in $\left\{z \in \mathbb{C}:|z|>\rho\right.$ and $\left.D_{\mathrm{e}}(z) \neq 0\right\}$ there exists $m=m(K) \in \mathbb{N}$ such that for $z \in K$, we have that $\Phi_{n}(z) \neq 0$ for all $n \geq m$.

Furthermore, each zero $\zeta$ of $D_{\mathrm{e}}$ in $\rho<|z|<1$ attracts as many zeros of $\Phi_{n}$ as its multiplicity, which tend to $\zeta$ with a geometric rate.

As mentioned in the Introduction, formula (37) (or the equivalent formula (10) ) shows that in the analytic case the Szegö's exterior asymptotics can be continued analytically through $\mathbb{T}_{1}$ up to the critical circle $\mathbb{T}_{\rho}$ (cf. [NT89] or [Sim04a, $\left.\S 7.1\right]$ ). The rate of convergence is a consequence of (32). For instance, when $r<|z|<1 / r$, by (24) and (34)-(35),

$$
\left|\frac{\Phi_{n}(z)}{z^{n}}-\frac{D_{\mathrm{e}}(z)}{\tau}\right|=\left|\frac{D_{\mathrm{e}}(z)}{\tau}\left(\mathcal{E}_{n}(z)-1\right)-\frac{\tau}{D_{\mathrm{i}}(z)} \mathcal{I}_{n}(z)\right| \leq \frac{C}{(1 / r-|z|)(|z|-r)}\left|\frac{r}{z}\right|^{n},
$$

where $C$ depends on $r$ and $\Lambda$ only.

The second statement of the Proposition is a direct application of this fact and of the Hurwitz's theorem.

Regarding the zeros of $\Phi_{n}$, we see that besides those matching the zeros of $D_{\text {e }}$ outside $\mathbb{T}_{\rho}$, the rest accumulates on $\overline{\mathbb{D}}_{\rho}$. Nevai-Totik's theorem [NT89] states that in fact $\mathbb{T}_{\rho}$ separates the regions of uniformly bounded and unbounded number of zeros of $\Phi_{n}$; more precisely,

$$
\rho=\inf \left\{r>0: \exists N(r)<\infty \text { so that } \forall n, \Phi_{n}(z) \text { has } \leq N(r) \text { zeros in }|z|>r\right\}
$$

(see also [Sim04a, §7.1]). Furthermore, by (32),

$$
\Phi_{n}(z)=-\frac{\tau}{D_{\mathrm{i}}(z)}\left(f_{n}^{(1)}(z)+h_{n}^{(1)}(z)\right), \quad z \in \mathbb{D}_{r},
$$

and from (34) - (35) it follows that there exists a constant $C$, depending only on $\Lambda$ and $r$, such that if $\rho<r^{\prime}<r$, then

$$
\left|h_{n}^{(1)}(z)\right| \leq \frac{C}{r-r^{\prime}} r^{3 n}, \quad z \in \mathbb{D}_{r^{\prime}} .
$$

As pointed out above, in a generic case the vast majority of zeros distributes uniformly at the critical circle $\mathbb{T}_{\rho}$, although some of them can remain inside, and by (38)-(39), stay close to the zeros of $f_{n}^{(1)}$ in $\mathbb{D}_{\rho}$.

Let us make these statements more precise. For that purpose we must impose some additional conditions on our weight $w$ (or function $\mathcal{F}$ ).

Proposition 2 Assume that there exists $0 \leq \rho^{\prime}<\rho$ such that $D_{\mathrm{e}}$ can be continued to the exterior of the circle $\mathbb{T}_{\rho^{\prime}}$, as an analytic function whose only singularities are on the circle $\mathbb{T}_{\rho}$, and these are all isolated. Denote by $a_{1}, \ldots, a_{u}$ the singularities (whose number is finite) of $D_{\mathrm{e}}$ on $\mathbb{T}_{\rho}$,

$$
\left|a_{1}\right|=\cdots=\left|a_{u}\right|=\rho .
$$


Then for $\rho<r^{\prime}<r$ there exist constants $0 \leq \delta=\delta\left(r^{\prime}\right)<1$ and $C=C\left(r^{\prime}\right)<+\infty$ such that for $\rho^{\prime}<|z| \leq r^{\prime}$ and $n \in \mathbb{N}$,

$$
\left|\Phi_{n}(z)-z^{n} \frac{D_{\mathrm{e}}(z)}{\tau}-\frac{1}{\tau D_{\mathrm{i}}(z)} \sum_{k=1}^{u} \operatorname{res}_{t=a_{k}}\left(\mathcal{F}(t) \frac{t^{n}}{t-z}\right)\right| \leq C\left(\rho^{n} \delta^{n}+r^{3 n}\right) .
$$

Furthermore, for every compact set $K \subset \mathbb{D}_{\rho}$ there exist constants $0 \leq \delta=\delta(K)<1$ and $C=C(K)<+\infty$ such that for $z \in K$,

$$
\left|\Phi_{n}(z)-\frac{1}{\tau D_{\mathrm{i}}(z)} \sum_{k=1}^{u} \operatorname{res}_{t=a_{k}}\left(\mathcal{F}(t) \frac{t^{n}}{t-z}\right)\right| \leq C\left(\rho^{n} \delta^{n}+\rho^{3 n}(1+\varepsilon)^{3 n}\right) .
$$

Remark 5 If $\mathcal{F}$ can be continued as an analytic function with a finite number of isolated singularities to whole disc $\mathbb{D}_{1}$, then we may take $\delta=0$ in the right hand sides in (41)-(42). Otherwise the right hand side in (42) may be replaced by an estimate of the form $C \rho^{n} \delta^{n}$.

Remark 6 This Proposition tells us that in general all the relevant information for the asymptotics of $\Phi_{n}$ 's in $\mathbb{D}_{r}$ comes from the singularities of the exterior Szegö function $D_{\mathrm{e}}$ on $\mathbb{T}_{\rho}$ (that is, from the first singularities of $D_{\mathrm{e}}$ we meet continuing it analytically inside the unit disc).

Remark 7 In order to isolate the zeros of $\Phi_{n}$, one must be able to analyze the approximation to $\Phi_{n}$ afforded by (41) and (42). For example, zero-free regions may be determined by (i) establishing zero-free regions for the approximation, and (ii) bounding $\Phi_{n}$ away from zero using the error estimates. Similarly, isolating the zeros can be done by first isolating the zeros of the approximation, and then using a Rouche' type argument for $\Phi_{n}$.

Remark 8 It is interesting to note that Theorem 1 provides global asymptotics for $\Phi_{n}$, and as a consequence, Proposition 2 above provides an asymptotic description which is valid even in vicinities of the poles $a_{k}$.

Remark 9 It is an interesting challenge to find general assumptions concerning the measure of orthogonality that lead to an effective characterization of the asymptotic behavior of the zeros of $\Phi_{n}$. Simon Sim04b, Sim04c] has placed assumptions on the Verblunsky coefficients which imply that all $a_{k}$ 's are simple poles. In this case the approximation afforded by (41) and (42) is completely explicit, and it is then straightforward (albeit technically complicated) to isolate and provide a complete asymptotic description for each and every zero of $\Phi_{n}$. While we could carry out this extension of [Sim04b, Sim04c], we rather choose to investigate a more general class of measures of orthogonality than considered in Sim04c], and demonstrate how it is possible to obtain the same detailed level of asymptotic information concerning the zeros of $\Phi_{n}$. The first result in this direction is the following Theorem. 
Definition 1 Let $a,|a|<1$, be a pole of a function $f$ analytic in $|z|<1$. We denote by mult $_{z=a} f(z)$ its multiplicity and say that $a$ is a dominant pole of $f$ if for any other singularity $b$ of $f$, either $|a|>|b|$ or $|a|=|b|$, but then $b$ is also a pole and

$$
\operatorname{mult}_{z=a} f(z) \geq \operatorname{mult}_{z=b} f(z) .
$$

In the sequel we use the following notation: for $a \in \mathbb{C}$ and $\varepsilon>0$,

$$
B_{\varepsilon}(a) \stackrel{\text { def }}{=}\{z \in \mathbb{C}:|z-a|<\varepsilon\} .
$$

Theorem 3 Assume that there exists $0 \leq \rho^{\prime}<\rho$ such that $D_{\mathrm{e}}$ can be continued to the exterior of the circle $\mathbb{T}_{\rho^{\prime}}$, as a meromorphic function whose only singularities are on the circle $T_{\rho}$. Denote by $a_{1}, \ldots, a_{u}$ the poles (whose number is finite) of $D_{\mathrm{e}}$ on $\mathbb{T}_{\rho}$, and assume that the dominant poles of $D_{\mathrm{e}}$ are $a_{1}, \ldots, a_{\ell}, \ell \leq u$, and their multiplicity is $m$.

Let $\varepsilon>0$. Then for $\rho^{\prime}<|z| \leq r-\varepsilon, z \notin \cup_{k=1}^{u} B_{\varepsilon}\left(a_{k}\right)$, and $n \in \mathbb{N}$,

$$
\Phi_{n}(z)=\frac{D_{\mathrm{e}}(z)}{\tau} z^{n}+\frac{1}{\tau D_{\mathrm{i}}(z)} \sum_{k=1}^{\ell}\left(\begin{array}{c}
n \\
m-1
\end{array}\right) a_{k}^{n-m+1} \frac{D_{\mathrm{i}}\left(a_{k}\right) \widehat{D}_{\mathrm{e}}\left(a_{k}\right)}{a_{k}-z}+h_{n}(z),
$$

where $\widehat{D}_{\mathrm{e}}\left(a_{k}\right)=\lim _{z \rightarrow a_{k}} D_{\mathrm{e}}(z)\left(z-a_{k}\right)^{m}, k=1, \ldots, \ell$. There exist a constant $0<C<+\infty$ independent of $n$ and $\varepsilon$, and a constant $0<\delta=\delta(\varepsilon)<1$, such that

$$
\left|h_{n}(z)\right| \leq \begin{cases}C\left(\rho^{n} \delta^{n}+r^{3 n}\right), & \text { if } m=1, \\ \frac{C}{\varepsilon^{m-1}} n^{m-2} \rho^{n}, & \text { if } m \geq 2 .\end{cases}
$$

Furthermore, for every compact set $K \subset \mathbb{D}_{\rho}$ there exists a constant $C=C(K)<\infty$ such that for $z \in K$, and $n \in \mathbb{N}$,

$$
\left|\frac{\tau D_{\mathrm{i}}(z)}{a_{1}^{n-m+1}}\left(\begin{array}{c}
n \\
m-1
\end{array}\right)^{-1} \Phi_{n}(z)-\sum_{k=1}^{\ell} \frac{D_{\mathrm{i}}\left(a_{k}\right) \widehat{D}_{\mathrm{e}}\left(a_{k}\right)}{a_{k}-z} e^{2 \pi i(n-m+1) \theta_{k}}\right| \leq \begin{cases}C \delta^{n}, & \text { if } m=1 \\
\frac{C}{n}, & \text { if } m \geq 2\end{cases}
$$

where

$$
\theta_{1}=1, \quad \text { and } \quad \theta_{k}=\frac{1}{2 \pi}\left(\arg a_{k}-\arg a_{1}\right), \quad k=2, \ldots, \ell .
$$

In particular, on every compact set $K \subset \mathbb{D}_{\rho}$, for all sufficiently large $n$ polynomials $\Phi_{n}$ can have at most $\ell-1$ zeros, counting their multiplicities.

Remark 10 In the spirit of Proposition 2 the asymptotics of $\Phi_{n}$ 's is governed by the dominant poles of $\mathcal{F}$ only. However, the error term $h_{n}$ in (45) is small only away from all poles $a_{k}$.

Observe that this result is applicable to weights of the form $w(z)=|R(z) S(z)|^{2}, z \in \mathbb{T}_{1}$, where $R$ is a rational function with at least one zero on $\mathbb{T}_{\rho}$ (or one pole on $\mathbb{T}_{1 / \rho}$ ), and $S$ is any function holomorphic and $\neq 0$ in any annulus, containing $\{\rho \leq|z| \leq 1 / \rho\}$. 
Remark 11 As mentioned in Remark 8 above, we may also obtain an asymptotic description of $\Phi_{n}$ in a vicinity of each of the poles $a_{k}$, and describe the behavior of the zeros near these singular points. We leave this exercise to the interested reader.

On the other hand, to investigate new phenomena, we consider in Section 5 the situation that there is a single isolated singularity of $D_{\mathrm{e}}$ on the circle of radius $\rho$ which is an essential singularity. For this case, we do carry out the detailed analysis of $\Phi_{n}$ near the location of the essential singularity.

Corollary 3 Under assumptions of Theorem 3, the Verblunsky coefficients satisfy

$$
\alpha_{n}=-\sum_{k=1}^{\ell}\left(\begin{array}{c}
n+1 \\
m-1
\end{array}\right) \overline{a_{k}^{n-m+1} D_{\mathrm{i}}\left(a_{k}\right) \widehat{D}_{\mathrm{e}}\left(a_{k}\right)}+ \begin{cases}\mathcal{O}\left(\rho^{n} \delta^{n}\right), & \text { if } m=1, \\
\mathcal{O}\left(n^{m-2} \rho^{n}\right), & \text { if } m \geq 2 .\end{cases}
$$

Remark 12 The case of Verblunsky coefficients with the asymptotics (47) and $m=1$ was analyzed in $[\operatorname{Sim04c}$. The subcase $m=1, u=1$ has been thoroughly studied in Sim04b].

Let us discuss now how Theorem 3 reveals the behavior of the zeros of the polynomials in $\mathbb{D}_{r}$. We distinguish two cases: the zeros approaching the critical circle $\mathbb{T}_{\rho}$ and those staying inside $\mathbb{D}_{\rho}$. Under assumptions of Theorem 3 let us denote

$$
G_{n}(z) \stackrel{\text { def }}{=} \sum_{k=1}^{\ell} \frac{D_{\mathrm{i}}\left(a_{k}\right) \widehat{D}_{\mathrm{e}}\left(a_{k}\right)}{a_{k}-z} e^{2 \pi i(n-m+1) \theta_{k}}
$$

$\theta_{k}$ defined in (46). Numerical experiments show that that the zeros of $\Phi_{n}$ approaching $\mathbb{T}_{\rho}$ distribute radially uniformly, with gaps ("missing zeros") at the poles $a_{k}$ and at possible zeros of $G_{n}$ on $\mathbb{T}_{\rho}$. We will use the following notation: for a given analytic function $f$ and for $\varepsilon>0$,

$$
\mathcal{Z}(f) \stackrel{\text { def }}{=}\{z: f(z)=0\}, \quad \mathcal{Z}_{\varepsilon}(f) \stackrel{\text { def }}{=} \bigcup_{z \in \mathcal{Z}(f)} B_{\varepsilon}(z)
$$

Also let

$$
\mathcal{B}(\varepsilon) \stackrel{\text { def }}{=}\left\{z: \rho^{\prime}+\varepsilon<|z|<r-\varepsilon, z \notin \cup_{k=1}^{u} B_{\varepsilon}\left(a_{k}\right) \cup \mathcal{Z}_{\varepsilon}\left(D_{\mathrm{e}}\right)\right\} .
$$

Theorem 4 Let $\varepsilon>0$, and assume that the hypotheses of Theorem 3 are satisfied. Put

$$
v_{n} \stackrel{\text { def }}{=} \rho\left(\begin{array}{c}
n \\
m-1
\end{array}\right)^{1 / n}
$$

Then there is a $\delta>0$ such that for all sufficiently large $n \in \mathbb{N}$ every pie slice of the form

$$
\left\{z: v_{n}(1-\delta / 2)<|z|<v_{n}(1+\delta / 2), \frac{\alpha+2 k_{1} \pi}{n}<\arg (z)<\frac{\alpha+2 k_{2} \pi}{n}\right\} \subset \mathcal{B}(\varepsilon) \backslash \mathcal{Z}_{\varepsilon}\left(G_{n}\right),
$$


with appropriately chosen $\alpha \in \mathbb{R}$, contains exactly $k=k_{2}-k_{1}$ zeros of $\Phi_{n}, z_{1}^{(n)}, \ldots, z_{k}^{(n)}$, satisfying

$$
\left|z_{i}^{(n)}\right|=\rho\left(1+\frac{1}{n} \log \left(\begin{array}{c}
n \\
m-1
\end{array}\right)+\mathcal{O}\left(\frac{1}{n}\right)\right)
$$

and

$$
\arg \left(z_{i+j}^{(n)}\right)-\arg \left(z_{i}^{(n)}\right)=\frac{2 \pi j}{n}+\mathcal{O}\left(\frac{1}{n^{2}}\right)
$$
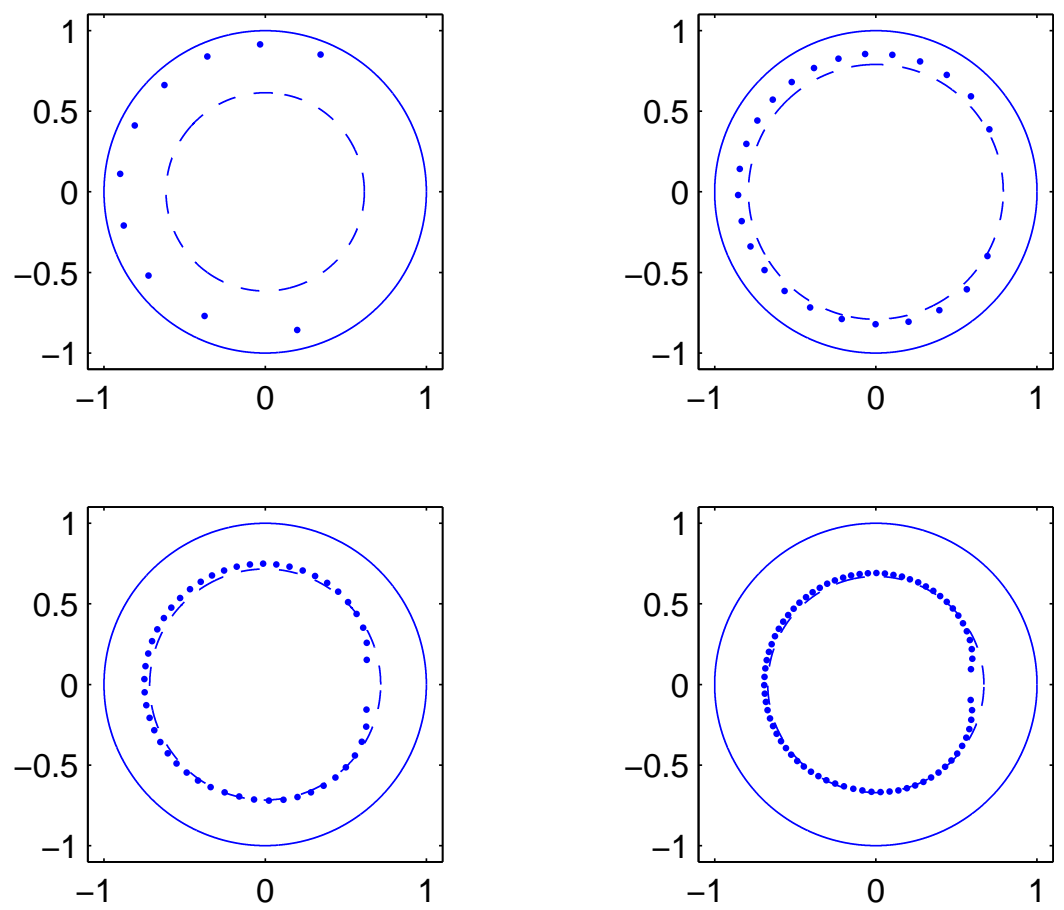

Figure 1: Zeros of $\Phi_{n}$ for $n=10,25,50,75$ (from left to right and from top to bottom) with $w(z)=\left|(z-1 / 2)^{10}\left(z-\zeta_{1} / 2\right)^{3}\right|^{2}, z \in \mathbb{T}_{1}$, where $\zeta_{1}=\exp (\pi i \sqrt{2})$. For comparison with the prediction (51) we plot in each case with a dashed line the circle of radius $\frac{1}{2}\left(1+\frac{1}{n} \log \left(\begin{array}{c}n \\ m-1\end{array}\right)\right)$.

Remark 13 We may consider an example for the situation described in Theorem 3, taking $w(z)=\left|(z-1 / 2)^{10}\left(z-\zeta_{1} / 2\right)^{3}\right|^{2}, z \in \mathbb{T}_{1}$, with $\zeta_{1}=\exp (\pi i \sqrt{2})$. The results of the numerical experiments appear in Fig. 1. Observe how the zeros exhibit the equidistribution pattern 
close to the circle of radius $0.5\left(1+\frac{1}{n} \log \left(\begin{array}{c}n \\ m-1\end{array}\right)\right)$ predicted by Theorem 4 , and basically "ignoring" the non-dominant pole $\zeta_{1} / 2$ of $\mathcal{F}$. An imaginative reader might observe though that the the influence of the non-dominant pole is not totally negligible: Fig. 1 shows a slight displacement of the zeros of $\Phi_{n}$ towards the origin in a neighborhood of $\zeta_{1} / 2$.

We can also be more precise about the accumulation set of zeros of $\Phi_{n}$ 's on compact sets of $\mathbb{D}_{\rho}$. Let

$$
Z \stackrel{\text { def }}{=} \bigcap_{k \geq 1} \overline{\bigcup_{n \geq k} \mathcal{Z}\left(\Phi_{n}\right)}
$$

Asymptotics (45) shows that the structure of $Z \cap \mathbb{D}_{\rho}$ depends on the relative positions of $a_{1}, \ldots, a_{\ell}$ on $\mathbb{T}_{\rho}$. Without loss of generality we may assume that $\theta_{1}=1, \theta_{2}, \ldots, \theta_{v}$ is the maximal subset of $\left\{\theta_{1}, \ldots, \theta_{\ell}\right\}$ linearly independent over the rational numbers $\mathbb{Q}$. Then there exist unique $r_{k j} \in \mathbb{Q}, k=1, \ldots, \ell, j=1, \ldots, v$, such that

$$
\theta_{k}=\sum_{j=1}^{v} r_{k j} \theta_{j}, \quad k=1, \ldots, \ell,
$$

(obviously, $r_{k j}=\delta_{k j}$, for $1 \leq k \leq v$ ). Furthermore, we may always take $r_{k 1} \geq 0, k=1, \ldots, \ell$. Denote

$$
r_{k 1}=\frac{p_{k}}{q_{k}}, \quad 0 \leq p_{k} \leq q_{k} \in \mathbb{Z}, \quad k=1, \ldots, \ell .
$$

Proposition 3 Let $t \in Z \cap \mathbb{D}_{\rho}$. If $v=1$ (that is, if all $\theta_{k} \in \mathbb{Q}$ ), then there exist $0 \leq s_{k}<q_{k}$, $s_{k} \in \mathbb{Z}, k=1, \ldots, \ell$ such that

$$
\sum_{k=1}^{\ell} \frac{D_{\mathrm{i}}\left(a_{k}\right) \widehat{D}_{\mathrm{e}}\left(a_{k}\right)}{a_{k}-t} e^{2 \pi i s_{k} / q_{k}}=0 .
$$

In this case $Z \cap \mathbb{D}_{\rho}$ is a finite set.

If $v \geq 2$, then additionally there exist $X_{2}, \ldots, X_{v} \in \mathbb{R}$ such that

$$
\sum_{k=1}^{\ell} \frac{D_{\mathrm{i}}\left(a_{k}\right) \widehat{D}_{\mathrm{e}}\left(a_{k}\right)}{a_{k}-t} e^{2 \pi i\left(s_{k} / q_{k}+\sum_{j=2}^{v} r_{k j} X_{j}\right)}=0 .
$$

Remark 14 As it was observed by Szabados [Sza79] (in the polynomial case), for $v=2$ equation (55) describes an algebraic curve of degree $\leq \ell$. In particular, if $\ell=2, Z \cap \mathbb{D}_{\rho}$ is a circular arc or a diameter in $\mathbb{D}_{\rho}$ (see Figure 2, left). For $v>2$ equation (55) describes a two-dimensional domain bounded by algebraic curves (cf. Figure 2, right). Observe that we plot all the zeros of $\Phi_{n}$ for $n=5, \ldots, 75$, in order to reveal the structure of $Z$ inside $\mathbb{T}_{1 / 2}$. 

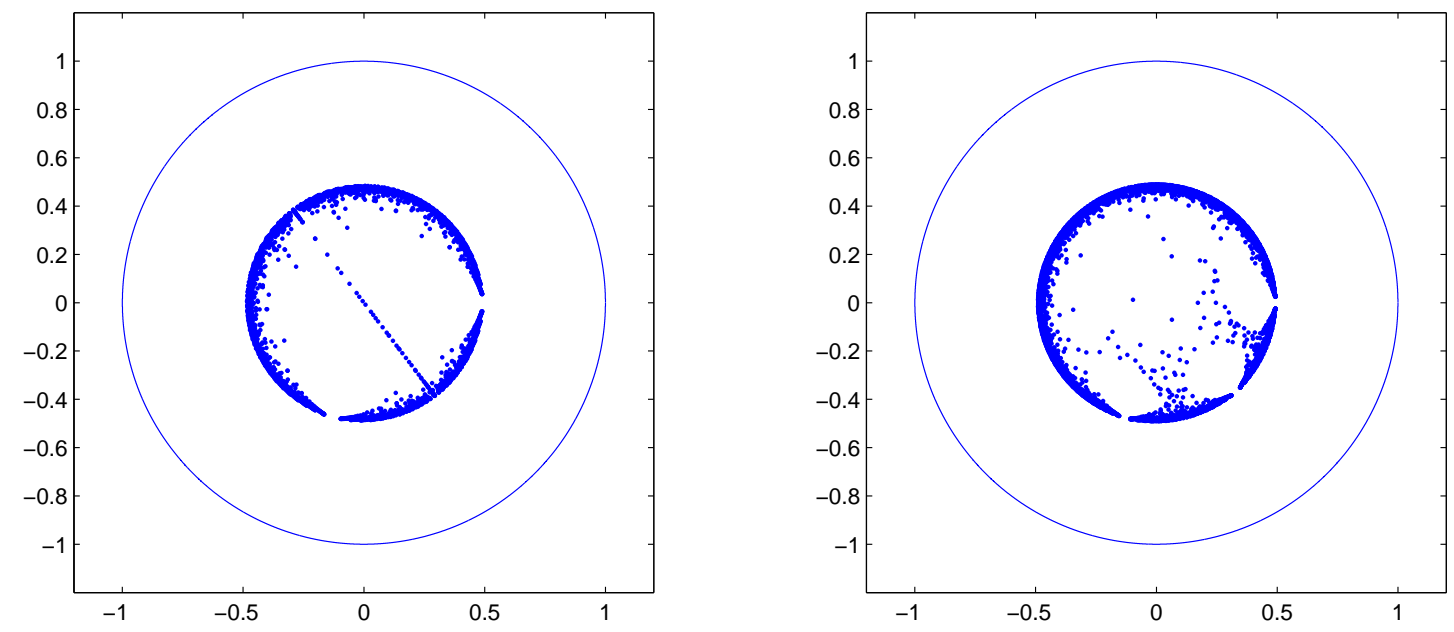

Figure 2: Zeros of $\Phi_{n}$ for $n=5, \ldots, 75$ with $w(z)=\left|(z-1 / 2)\left(z-\zeta_{1} / 2\right)\right|^{2}$ (left) and $w(z)=$ $\left|(z-1 / 2)\left(z-\zeta_{1} / 2\right)\left(z-\zeta_{2} / 2\right)\right|^{2}$ (right), $z \in \mathbb{T}_{1}$, where $\zeta_{1}=\exp (\pi i \sqrt{2}), \zeta_{2}=\exp (\pi i \sqrt{3})$.

We can be more specific in the case of a rational weight, i.e. when $w$ has the following form:

$$
w(z)=|r(z)|^{2}, \quad|z|=1,
$$

where $r$ is a rational function non vanishing on $\mathbb{T}_{1}$; we may write

$$
r(z)=\frac{p_{\mathrm{i}}}{q_{\mathrm{i}}}(z) \frac{p_{\mathrm{e}}}{q_{\mathrm{e}}}(z)
$$

where $p_{\mathrm{i}}$ and $q_{\mathrm{i}}$ are polynomials without zeros in $|z| \leq 1, p_{\mathrm{e}}$ and $q_{\mathrm{e}}$ are polynomials without zeros in $|z| \geq 1$, and $p_{\mathrm{e}}(0) \neq 0$ and $q_{\mathrm{e}}(0) \neq 0$. Then,

$$
|r(z)|=\left|\frac{p_{\mathrm{i}}}{q_{\mathrm{i}}}(z) \frac{p_{\mathrm{e}}}{q_{\mathrm{e}}}(z)\right|=\left|e^{i \alpha} \frac{p_{\mathrm{i}}}{q_{\mathrm{i}}}(z) \frac{p_{\mathrm{e}}}{q_{\mathrm{e}}}(z) \frac{p_{\mathrm{i}}^{*}}{p_{\mathrm{i}}}(z) \frac{q_{\mathrm{i}}}{q_{\mathrm{i}}^{*}}(z)\right|=\left|e^{i \alpha} \frac{p_{\mathrm{i}}^{*}}{q_{\mathrm{i}}^{*}}(z) \frac{p_{\mathrm{e}}}{q_{\mathrm{e}}}(z)\right|=|R(z)|, \quad \alpha \in \mathbb{R},
$$

where $R$ has neither zeros nor poles in $|z| \geq 1$; since in this identity we may multiply $R$ by any power of $z$ and any constant with absolute value equal to 1 , without loss of generality we may assume that $0<R(\infty)<\infty$.

Definition 2 We say that $w(z)=|R(z)|^{2},|z|=1$ is a normalized representation of the rational weight $w$ if $R$ is a rational function with neither zeros nor poles in $|z| \geq 1$ and such that $0<R(\infty)<\infty$. 
If $w(z)=|R(z)|^{2},|z|=1$, is a normalized representation of $w$, by rewriting this identity on $\mathbb{T}_{1}$ as

$$
w(z)=|R(z)|^{2}=R(z) \overline{R(z)}=R(z) \bar{R}(1 / z),
$$

we immediately obtain that in this case,

$$
D_{\mathrm{i}}(z)=\bar{R}(1 / z), \quad D_{\mathrm{e}}(z)=\frac{1}{R(z)}, \quad \tau=\frac{1}{R(\infty)} .
$$

Furthermore,

$$
\mathcal{F}(z)=\frac{\bar{R}(1 / z)}{R(z)}
$$

is a Blaschke product with its poles in $|z|<1$ coinciding with the zeros of $R$, and with its zeros in $|z|<1$ matching the poles of the same functions.

Let $a_{1}, \ldots, a_{\ell}$ be the zeros of $R$ in $|z|<1$. Now by (32),

$$
\Phi_{n}(z)=z^{n} \frac{R(\infty)}{R(z)}+\frac{R(\infty)}{\bar{R}(1 / z)} \sum_{k=1}^{\ell} \operatorname{res}_{t=a_{k}}\left(\frac{\bar{R}(1 / t)}{R(t)} \frac{t^{n}}{t-z}\right)+h_{n}(z), \quad|z|<1,
$$

where by (34)-(35), $\left|h_{n}(z)\right| \leq C /(r-|z|) r^{3 n}$, being $C$ a constant depending on $\Lambda$ and $r$ only.

In particular, if all the zeros of $R$ in $|z|<1$ are simple, we get

$$
\Phi_{n}(z)=z^{n} \frac{R(\infty)}{R(z)}+\frac{R(\infty)}{\bar{R}(1 / z)} \sum_{k=1}^{\ell} \frac{\bar{R}\left(1 / a_{k}\right)}{R^{\prime}\left(a_{k}\right)} \frac{a_{k}^{n}}{a_{k}-z}+h_{n}(z), \quad|z|<1 .
$$

Remark 15 As mentioned in the introduction, Szabados Sza79 obtained a formula similar to (60) for the case of a polynomial weight; he also observed the equidistribution of the zeros approaching the critical circle $\mathbb{T}_{\rho}$. Rational weights with one dominant singularity have been discussed also by Ismail and Ruedemann in IR92.

Remark 16 To contrast some of these results with a case of a more severe singularity, in Section 5 we consider the weight of orthogonality

$$
w(t)=w_{1}(t)=\left|\exp \left(\frac{1}{a-t}\right)\right|^{2}, \quad t \in \mathbb{T}_{1},
$$

with $0<a<1$, as well as its inverse, $1 / w$. For $w$ in (61) the asymptotics of the Verblunsky coefficients is

$$
\alpha_{n}=-\frac{1}{2 \sqrt{\pi}} t_{+}^{n} \mathcal{F}\left(t_{+}\right)\left(\frac{a}{n}\right)^{3 / 4}\left(1+\mathcal{O}\left(\frac{1}{n^{1 / 2}}\right)\right), \quad n \rightarrow \infty
$$


(see Eq. (105)), where $t_{+}$is the solution of the equation

$$
\frac{1}{t}-\frac{1}{n+1}\left(\frac{t}{a t-1}+\frac{1}{t-a}\right)=0
$$

satisfying

$$
t_{+}=a+\sqrt{\frac{a}{n+1}}+\mathcal{O}\left(\frac{1}{n}\right), \quad n \rightarrow \infty,
$$

where we take the positive square root.

For the weight $1 / w$, with $w$ defined in (61), the asymptotics of the Verblunsky coefficients is

$$
\alpha_{n}=-\frac{1}{\sqrt{\pi}}\left(\frac{a}{n}\right)^{3 / 4} \operatorname{Re}\left(t_{+}^{n} \mathcal{F}\left(t_{+}\right)\right)\left(1+\mathcal{O}\left(\frac{1}{n^{1 / 2}}\right)\right), \quad n \rightarrow \infty,
$$

(see Eq. (105) ), but $t_{+}$is now the solution of the equation

$$
\frac{1}{t}+\frac{1}{n+1}\left(\frac{t}{a t-1}+\frac{1}{t-a}\right)=0
$$

satisfying

$$
t_{+}=a+i \sqrt{\frac{a}{n+1}}+\mathcal{O}\left(\frac{1}{n}\right), \quad n \rightarrow \infty,
$$

where we take again the positive square root.
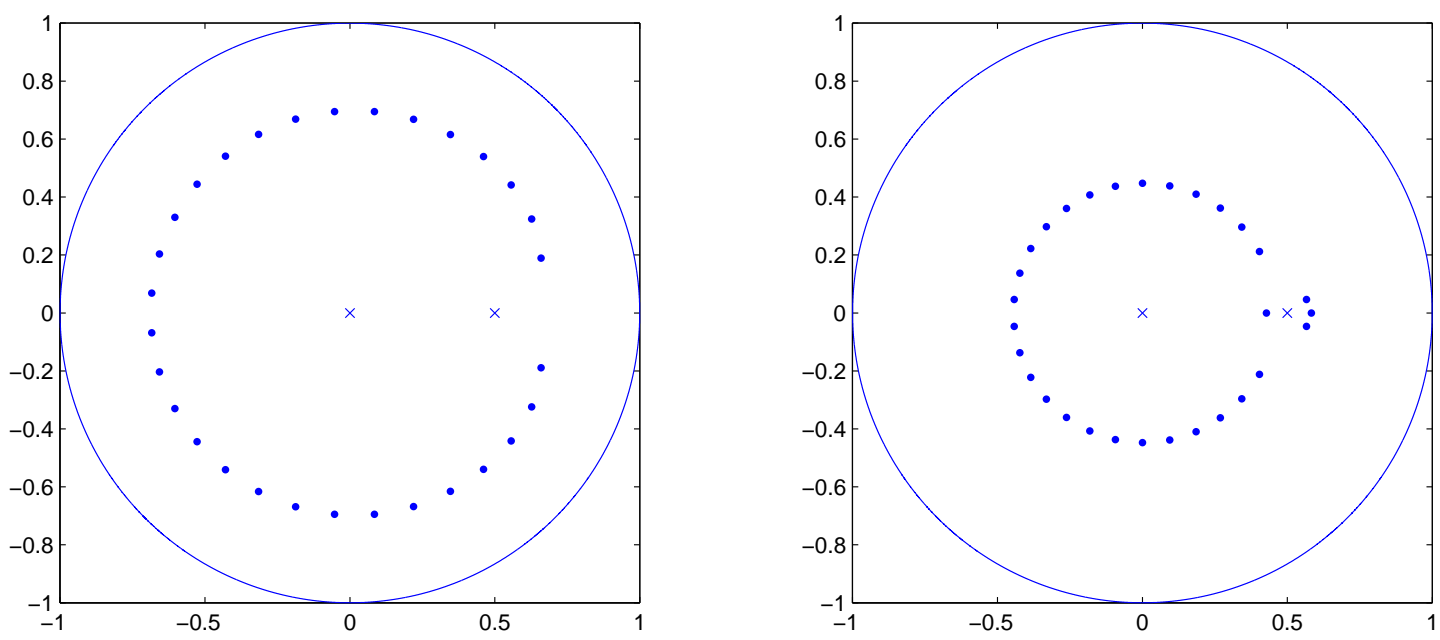

Figure 3: Zeros of $\Phi_{30}$ for weights $w$ (left) and 1/w (right), with $w$ given in (61) and $a=1 / 2$. 
Also the zero behavior in both cases is different. In order to entice the reader we show in Fig. 3 images of the zeros of $\Phi_{n}$ (for $n=30$ and $a=1 / 2$ ), whose complete asymptotic description appears in Section 5 .

\section{Steepest descent analysis for orthogonal polynomials}

Observe that from the orthogonality conditions (11) it follows that

$$
\oint_{\mathbb{T}_{1}} \varphi_{n}(z) \overline{z^{k}} w(z)|d z|=\frac{1}{i} \oint_{\mathbb{T}_{1}} \varphi_{n}(z) z^{n-k-1} \frac{w(z)}{z^{n}} d z=0 \quad \text { for } k=0,1, \ldots, n-1
$$

The reversed polynomials $\varphi^{*}(z)=z^{n} \overline{\varphi_{n}}(1 / z)$ also satisfy the following orthogonality conditions:

$$
\oint_{\mathbb{T}_{1}} \varphi_{n-1}^{*}(z) z^{k} \frac{w(z)}{z^{n}} d z=i \oint_{\mathbb{T}_{1}} \overline{\varphi_{n-1}(z)} z^{k} w(z)|d z|= \begin{cases}0, & k=0,1, \ldots, n-2 \\ i / \kappa_{n-1}, & k=n-1\end{cases}
$$

Hence,

$$
Y(z)=\left(\begin{array}{cc}
\Phi_{n}(z) & \frac{1}{2 \pi i} \oint_{\mathbb{T}_{1}} \frac{\Phi_{n}(t) w(t) d t}{t^{n}(t-z)} \\
-2 \pi \kappa_{n-1} \varphi_{n-1}^{*}(z) & -\frac{\kappa_{n-1}}{i} \oint_{\mathbb{T}_{1}} \frac{\varphi_{n-1}^{*}(t) w(t) d t}{t^{n}(t-z)}
\end{array}\right)
$$

is a unique solution of the following Riemann-Hilbert problem: $Y$ is holomorphic in $\mathbb{C} \backslash \mathbb{T}_{1}$,

$$
Y_{+}(t)=Y_{-}(t)\left(\begin{array}{cc}
1 & w(t) / t^{n} \\
0 & 1
\end{array}\right), \quad z \in \mathbb{T}_{1}
$$

and

$$
\lim _{z \rightarrow \infty} Y(z)\left(\begin{array}{cc}
z^{-n} & 0 \\
0 & z^{n}
\end{array}\right)=I
$$

where $I$ is the $2 \times 2$ identity matrix. Observe also that

$$
\kappa_{n-1}^{2}=-\frac{Y_{21}(0)}{2 \pi}
$$

(see [BDJ99] or [Dei99a] for more details on the Riemann-Hilbert characterization of orthogonal polynomials).

Let us define

$$
H(z) \stackrel{\text { def }}{=}\left\{\begin{array}{cc}
\left(\begin{array}{cc}
z^{-n} & 0 \\
0 & z^{n}
\end{array}\right), & \text { if }|z|>1 \\
I, & \text { if }|z|<1
\end{array}\right.
$$


and put

$$
T(z) \stackrel{\text { def }}{=} Y(z) H(z)
$$

Then $T$ is holomorphic in $\mathbb{C} \backslash \mathbb{T}_{1}$,

$$
T_{+}(t)=T_{-}(t)\left(\begin{array}{cc}
t^{n} & w(t) \\
0 & t^{-n}
\end{array}\right), \quad t \in \mathbb{T}_{1}
$$

and

$$
\lim _{z \rightarrow \infty} T(z)=I
$$

Next transformation is induced by the following factorization of the jump matrix for $T$ :

$$
\left(\begin{array}{cc}
t^{n} & w(t) \\
0 & t^{-n}
\end{array}\right)=\left(\begin{array}{cc}
1 & 0 \\
1 /\left(t^{n} w(t)\right) & 1
\end{array}\right)\left(\begin{array}{cc}
0 & w(t) \\
-1 / w(t) & 0
\end{array}\right)\left(\begin{array}{cc}
1 & 0 \\
t^{n} / w(t) & 1
\end{array}\right) .
$$

Hence, with $r, \rho<r<1$, fixed above we determine the following regions (Figure 4):

$$
\begin{aligned}
\Omega_{0} & =\{z:|z|<r\}, \quad \Omega_{\infty}=\{z:|z|>1 / r\} \\
\Omega_{+} & =\{z: r<|z|<1\}, \quad \Omega_{-}=\{z: 1<|z|<1 / r\} .
\end{aligned}
$$

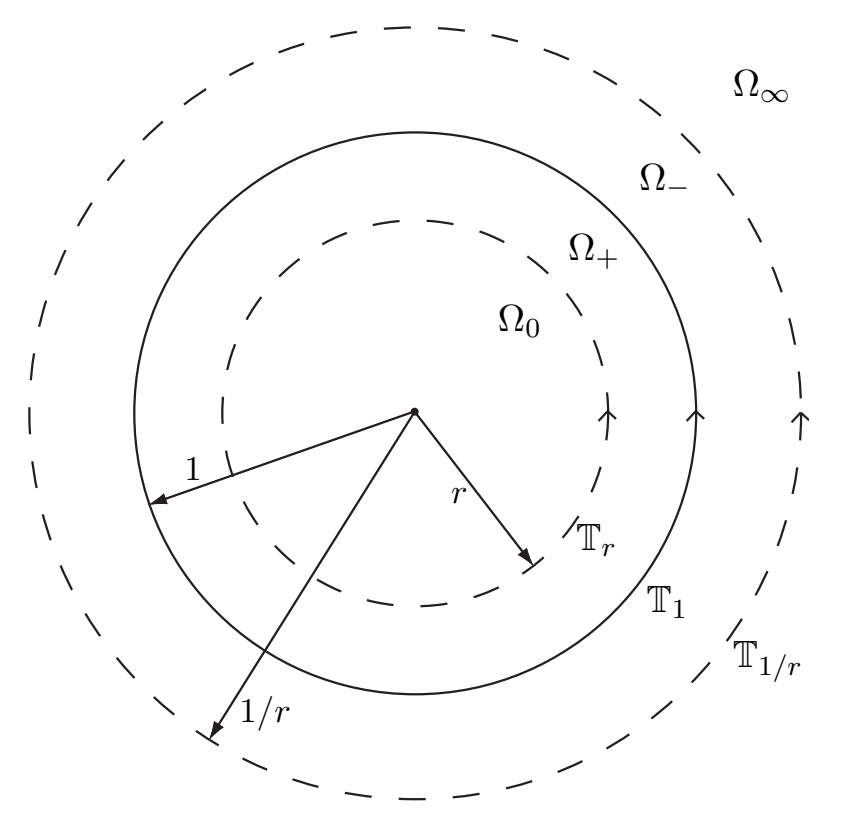

Figure 4: Opening lenses. 
We define now

$$
U(z) \stackrel{\text { def }}{=} T(z) K(z)
$$

where

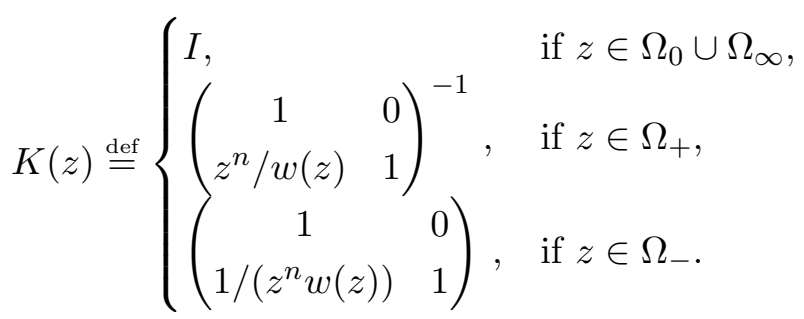

Then $U$ is holomorphic in $\mathbb{C} \backslash\left(\mathbb{T}_{r} \cup \mathbb{T}_{1} \cup \mathbb{T}_{1 / r}\right)$,

$$
\lim _{z \rightarrow \infty} U(z)=I
$$

and

$$
U_{+}(t)=U_{-}(t) J_{U}(t), \quad t \in\left(\mathbb{T}_{r} \cup \mathbb{T}_{1} \cup \mathbb{T}_{1 / r}\right),
$$

where

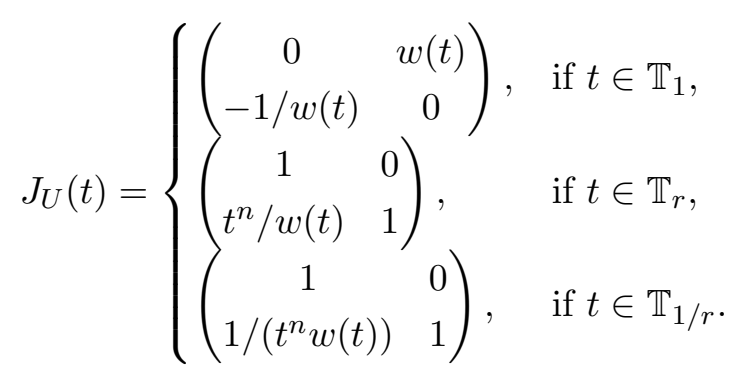

Using the piece-wise analytic matrix

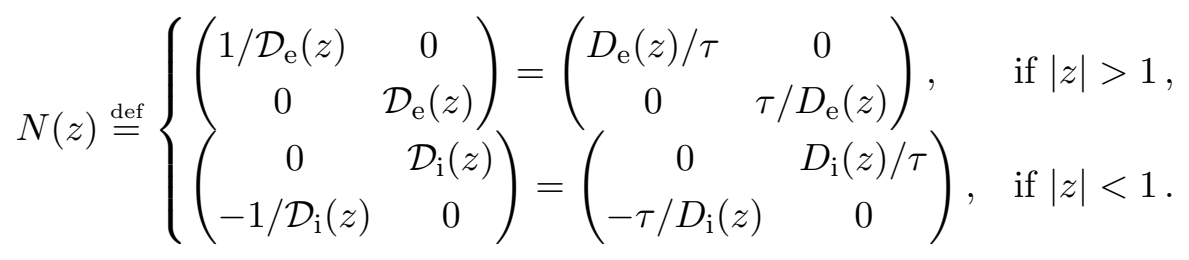

we make a new transformation, defining

$$
S(z) \stackrel{\text { def }}{=} U(z) N^{-1}(z) .
$$

Matrix $S$ is holomorphic in $\mathbb{C} \backslash\left(\mathbb{T}_{r} \cup \mathbb{T}_{1} \cup \mathbb{T}_{1 / r}\right), \lim _{z \rightarrow \infty} S(z)=I$, and

$$
S_{+}(t)=S_{-}(t) J_{S}(t), \quad t \in \mathbb{T}_{r} \cup \mathbb{T}_{1} \cup \mathbb{T}_{1 / r},
$$


where

$$
J_{S}(t)=N_{-} J_{U} N_{+}^{-1}= \begin{cases}I, & \text { if } t \in \mathbb{T}_{1}, \\
\left(\begin{array}{cc}
1 & -t^{n} \mathcal{D}_{\mathrm{i}}^{2}(t) / w(t) \\
0 & 1 \\
1 & 0 \\
\mathcal{D}_{\mathrm{e}}^{2}(t) /\left(t^{n} w(t)\right) & 1
\end{array}\right), & \text { if } t \in \mathbb{T}_{r}, \\
\text { if } t \in \mathbb{T}_{1 / r} .\end{cases}
$$

This shows in particular that $S$ is analytic across $\mathbb{T}_{1}$. Using the definition of $\mathcal{F}$, the jump matrix function $J_{S}$ can be rewritten as

$$
J_{S}(t)=N_{-} J_{U} N_{+}^{-1}= \begin{cases}I, & \text { if } t \in \mathbb{T}_{1}, \\
\left(\begin{array}{cc}
1 & -t^{n} \mathcal{F}(t) / \tau^{2} \\
0 & 1 \\
1 & 0 \\
\tau^{2} /\left(t^{n} \mathcal{F}(t)\right) & 1
\end{array}\right), & \text { if } t \in \mathbb{T}_{r}, \\
\text { if } t \in \mathbb{T}_{1 / r} .\end{cases}
$$

Observe that

$$
J_{S}(z)=I+\mathcal{O}\left(r^{n}\right), \quad z \in \mathbb{T}_{r} \cup \mathbb{T}_{1 / r} .
$$

Unravelling these transformations we have

$$
Y(z)=S(z) N(z) K^{-1}(z) H(z)
$$

where $H, K$ and $N$ are defined in (63), (66) and (67), respectively.

Proof of Theorem 1. In order to make formula (71) useful we need an asymptotic expression for $S$. It can be obtained if we observe that $S$ solves the following Riemann-Hilbert problem: $S_{+}=S_{-} J_{S}$ on $\mathbb{T}_{r} \cup \mathbb{T}_{1 / r}$, and $S(z)=I+\mathcal{O}(1 / z)$ as $z \rightarrow \infty$. The jump condition may be rewritten equivalently as

$$
(S-I)_{+}-(S-I)_{-}=S_{-}\left(J_{S}-I\right) \quad \text { on } \mathbb{T}_{r} \cup \mathbb{T}_{1 / r} .
$$

Let us introduce the following notation: for $f$ defined and continuous on $\mathbb{T}_{r} \cup \mathbb{T}_{1 / r}$,

$$
\mathcal{C}(f)(z) \stackrel{\text { def }}{=} \frac{1}{2 \pi i} \oint_{\mathbb{T}_{r} \cup \mathbb{T}_{1 / r}} \frac{f(t) d t}{t-z},
$$

is the Cauchy operator, and

$$
\mathcal{C}_{-}(f)(z) \stackrel{\text { def }}{=} \lim _{\substack{u \rightarrow \rightarrow \\ u \in \text { "“-" side }}} \mathcal{C}(f)(u), \quad \mathcal{C}_{+}(f)(z) \stackrel{\text { def }}{=} \lim _{\substack{u \rightarrow z \\ u \in \text { “+” side }}} \mathcal{C}(f)(u),
$$


are the Cauchy projection operators on the "-" and "+"-sides of the corresponding curves, respectively. Furthermore, let

$$
\mathcal{P}(f)(z) \stackrel{\text { def }}{=} \mathcal{C}\left(f\left(J_{S}-I\right)\right)(z), \quad \mathcal{P}_{-}(f)(z) \stackrel{\text { def }}{=} \mathcal{C}_{-}\left(f\left(J_{S}-I\right)\right)(z) .
$$

We can use the fact that Sokhotsky-Plemelj's formula $\mathcal{C}_{+}-\mathcal{C}_{-}=I$ is valid also in $L^{2}(\mathrm{cf}$. [Ste70] and Dei99a, §7.1]).

Then, due to the normalization at infinity, (72) can be expressed, equivalently, as

$$
S-I=\mathcal{C}\left(S_{-}\left(J_{S}-I\right)\right)=\mathcal{P}\left(S_{-}\right)=\mathcal{P}(I)+\mathcal{P}\left(S_{-}-I\right) .
$$

Considering the "-" boundary values in (73), we get that $\mu \stackrel{\text { def }}{=} S_{-}-I$ satisfies $\mu=\mathcal{P}_{-}(I)+$ $\mathcal{P}_{-}(\mu)$, or

$$
\left(I-\mathcal{P}_{-}\right)(\mu)=\mathcal{P}_{-}(I)
$$

This may be viewed as a singular integral equation for $\mu$. Since $\mathcal{C}_{-}$is a bounded linear operator in $L^{2}\left(\mathbb{T}_{r} \cup \mathbb{T}_{1 / r}\right)$, there exists a constant $0<A<+\infty$, such that $\left\|\mathcal{P}_{-} f\right\| \leq A\|f\|$ for every $f \in L^{2}\left(\mathbb{T}_{r} \cup \mathbb{T}_{1 / r}\right)$, where we use the $L^{2}$ norm. In particular, using (70) we get

$$
\left\|\mathcal{P}_{-}(f)\right\| \leq A\left\|f\left(J_{S}-I\right)\right\| \leq A\left\|J_{S}-I\right\|_{L^{\infty}}\|f\| \leq A r^{n}\|f\|,
$$

for $n$ sufficiently large. For these $n$ 's, $\left\|\mathcal{P}_{-}\right\|<1$, and we can solve (74) using Neumann series:

$$
\mu=\left(I-\mathcal{P}_{-}\right)^{-1}\left(\mathcal{P}_{-}(I)\right)=\sum_{k=0}^{\infty} \mathcal{P}_{-}^{k}\left(\mathcal{P}_{-}(I)\right)=\sum_{k=1}^{\infty} \mathcal{P}_{-}^{k}(I),
$$

or equivalently,

$$
S_{-}=\sum_{k=0}^{\infty} \mathcal{P}_{-}^{k}(I)
$$

Properly speaking, if we use the $L^{2}$ theory of Riemann-Hilbert problems, this series can be shown to converge in the $L^{2}$ sense. However, the series is, in fact, uniformly convergent. Indeed, straightforward contour deformations show that each successive integral in $\mathcal{P}_{-}^{k}(I)$ taken over a circle of radius $r$ may be deformed to a circle of radius $r^{\prime}$ with $r^{\prime}<r$, and similarly each successive integral taken over a circle of radius $1 / r$ may be deformed to a circle of radius $1 / r^{\prime}$ with $r^{\prime}<r$. Then arguments quite analogous to those leading to (24) may be used to estimate each term $\mathcal{P}_{-}^{k}(I)$, and the uniform convergence is evident.

Replacing this series in (73) we get the full asymptotic expansion for $S$ :

$$
S(z)=I+\mathcal{P}\left(S_{-}\right)=I+\mathcal{P}\left(\sum_{k=0}^{\infty} \mathcal{P}_{-}^{k}(I)\right)=I+\sum_{k=0}^{\infty} \mathcal{P}\left(\mathcal{P}_{-}^{k}(I)\right)=\sum_{k=0}^{\infty} S^{(k)}
$$


where

$$
S^{(0)}=I, \quad \text { and } \quad S^{(k+1)}=\mathcal{P}\left(S_{-}^{(k)}\right), \quad k \geq 0 .
$$

Using the explicit expression for $J_{S}$ we get

$$
S^{(1)}(z)=\frac{1}{2 \pi i} \oint_{\mathbb{T}_{r} \cup \mathbb{T}_{1 / r}} \frac{\left(J_{S}(t)-I\right) d t}{t-z}=\left(\begin{array}{cc}
0 & f_{n}^{(1)}(z) \\
g_{n}^{(1)}(z) & 0
\end{array}\right),
$$

where

$$
f_{n}^{(1)} \stackrel{\text { def }}{=} \mathcal{M}_{n}^{\mathrm{i}}(1), \quad g_{n}^{(1)} \stackrel{\text { def }}{=} \mathcal{M}_{n}^{\mathrm{e}}(1),
$$

and operators $\mathcal{M}_{n}^{\mathrm{i}}, \mathcal{M}_{n}^{\mathrm{e}}$ have been defined in (18)-(19). Furthermore,

$$
\begin{aligned}
S^{(2)}(z) & =\mathcal{P}\left(S_{-}^{(1)}\right)(z)=\frac{1}{2 \pi i} \oint_{\mathbb{T}_{r} \cup \mathbb{T}_{1 / r}} \frac{S_{-}^{(1)}(t)\left(J_{S}(t)-I\right) d t}{t-z} \\
& =\frac{1}{2 \pi i} \oint_{\mathbb{T}_{1 / r}}\left(\begin{array}{cc}
0 & \left(f_{n}^{(1)}\right)_{-}(t) \\
\left(g_{n}^{(1)}\right)_{-}(t) & 0
\end{array}\right)\left(\begin{array}{cc}
0 & 0 \\
\mathcal{D}_{\mathrm{e}}^{2}(t) /\left(t^{n} w(t)\right) & 0
\end{array}\right) \frac{d t}{t-z} \\
& +\frac{1}{2 \pi i} \oint_{\mathbb{T}_{r}}\left(\begin{array}{cc}
0 & \left(f_{n}^{(1)}\right)_{-}(t) \\
\left(g_{n}^{(1)}\right)_{-}(t) & 0
\end{array}\right)\left(\begin{array}{cc}
0 & -t^{n} \mathcal{D}_{\mathrm{i}}^{2}(t) / w(t) \\
0 & 0
\end{array}\right) \frac{d t}{t-z} \\
& =\frac{\tau^{2}}{2 \pi i} \oint_{\mathbb{T}_{1 / r}}\left(\begin{array}{cc}
1 & 0 \\
0 & 0
\end{array}\right) \\
& =\left(\begin{array}{cc}
f_{n}^{(2)}(z) & 0 \\
0 & g_{n}^{(2)}(z)
\end{array}\right),
\end{aligned}
$$

where

$$
g_{n}^{(2)} \stackrel{\text { def }}{=}\left(\mathcal{M}_{n}^{\mathrm{i}} \circ \mathcal{M}_{n}^{\mathrm{e}}\right)(1)=M_{n}^{\mathrm{i}}\left(g_{n}^{(1)}\right), \quad f_{n}^{(2)} \stackrel{\text { def }}{=}\left(\mathcal{M}_{n}^{\mathrm{e}} \circ \mathcal{M}_{n}^{\mathrm{i}}\right)(1)=M_{n}^{\mathrm{e}}\left(f_{n}^{(1)}\right) .
$$

Iterating this process, we get that

$$
S=\left(\begin{array}{cc}
1+\sum_{k=1}^{\infty} f_{n}^{(2 k)} & \sum_{k=0}^{\infty} f_{n}^{(2 k+1)} \\
\sum_{k=0}^{\infty} g_{n}^{(2 k+1)} & 1+\sum_{k=1}^{\infty} g_{n}^{(2 k)}
\end{array}\right),
$$

where the matrix entries are defined recursively by

$f_{n}^{(1)} \stackrel{\text { def }}{=} \mathcal{M}_{n}^{\mathrm{i}}(1), \quad f_{n}^{(2)} \stackrel{\text { def }}{=} \mathcal{M}_{n}^{\mathrm{e}}\left(f_{n}^{(1)}\right), \quad f_{n}^{(2 k+1)} \stackrel{\text { def }}{=} \mathcal{M}_{n}^{\mathrm{i}}\left(f_{n}^{(2 k)}\right), \quad f_{n}^{(2 k+2)} \stackrel{\text { def }}{=} \mathcal{M}_{n}^{\mathrm{e}}\left(f_{n}^{(2 k+1)}\right), \quad k \in \mathbb{N}$, 
and

$g_{n}^{(1)} \stackrel{\text { def }}{=} \mathcal{M}_{n}^{\mathrm{e}}(1), \quad g_{n}^{(2)} \stackrel{\text { def }}{=} \mathcal{M}_{n}^{\mathrm{i}}\left(g_{n}^{(1)}\right), \quad g_{n}^{(2 k+1)} \stackrel{\text { def }}{=} \mathcal{M}_{n}^{\mathrm{e}}\left(g_{n}^{(2 k)}\right), \quad g_{n}^{(2 k+2)} \stackrel{\text { def }}{=} \mathcal{M}_{n}^{\mathrm{i}}\left(g_{n}^{(2 k+1)}\right), \quad k \in \mathbb{N}$.

It is usually the case in the asymptotic analysis of Riemann-Hilbert problems that the expansion for the error matrix is, at the end of the day, an asymptotic expansion, rather than being uniformly convergent. However, in the present setting, estimates (24) and (33) show that the series appearing in (75) are actually uniformly convergent (at least, for $n$ satisfying (30) $)$ !

Now we may replace (75) in (171) in order to find an expression for $Y$. We must do it independently in each region.

In the domain $\Omega_{\infty}$,

$$
N(z)=\left(\begin{array}{cc}
D_{\mathrm{e}}(z) / \tau & 0 \\
0 & \tau / D_{\mathrm{e}}(z)
\end{array}\right), \quad K(z)=I, \quad H(z)=\left(\begin{array}{cc}
z^{-n} & 0 \\
0 & z^{n}
\end{array}\right) .
$$

Hence,

$$
Y(z)=S(z)\left(\begin{array}{cc}
z^{n} D_{\mathrm{e}}(z) / \tau & 0 \\
0 & \tau /\left(z^{n} D_{\mathrm{e}}(z)\right)
\end{array}\right)
$$

and

$$
\Phi_{n}(z)=Y_{11}(z)=S_{11}(z) z^{n} D_{\mathrm{e}}(z) / \tau=\frac{z^{n} D_{\mathrm{e}}(z)}{\tau}\left(1+\sum_{k=1}^{\infty} f_{n}^{(2 k)}(z)\right), \quad|z|>1 / r .
$$

In the domain $\Omega_{-}$,

$$
N(z)=\left(\begin{array}{cc}
D_{\mathrm{e}}(z) / \tau & 0 \\
0 & \tau / D_{\mathrm{e}}(z)
\end{array}\right), \quad K(z)=\left(\begin{array}{cc}
1 & 0 \\
1 /\left(z^{n} w(z)\right) & 1
\end{array}\right), \quad H(z)=\left(\begin{array}{cc}
z^{-n} & 0 \\
0 & z^{n}
\end{array}\right) .
$$

Hence,

$$
Y(z)=S(z)\left(\begin{array}{cc}
z^{n} D_{\mathrm{e}}(z) / \tau & 0 \\
-\tau / D_{\mathrm{i}}(z) & \tau /\left(z^{n} D_{\mathrm{e}}(z)\right)
\end{array}\right)
$$

and for $1 \leq|z|<1 / r$,

$$
\begin{aligned}
\Phi_{n}(z) & =Y_{11}(z)=S_{11}(z) \frac{z^{n} D_{\mathrm{e}}(z)}{\tau}-S_{12}(z) \frac{\tau}{D_{\mathrm{i}}(z)} \\
& =\frac{z^{n} D_{\mathrm{e}}(z)}{\tau}\left(1+\sum_{k=1}^{\infty} f_{n}^{(2 k)}(z)\right)-\frac{\tau}{D_{\mathrm{i}}(z)}\left(\sum_{k=0}^{\infty} f_{n}^{(2 k+1)}(z)\right) .
\end{aligned}
$$

In the domain $\Omega_{+}$,

$$
N(z)=\left(\begin{array}{cc}
0 & D_{\mathrm{i}}(z) / \tau \\
-\tau / D_{\mathrm{i}}(z) & 0
\end{array}\right), \quad K^{-1}(z)=\left(\begin{array}{cc}
1 & 0 \\
z^{n} / w(z) & 1
\end{array}\right), \quad H(z)=I .
$$


Hence,

$$
Y(z)=S(z)\left(\begin{array}{cc}
z^{n} D_{\mathrm{e}}(z) / \tau & D_{\mathrm{i}}(z) / \tau \\
-\tau / D_{\mathrm{i}}(z) & 0
\end{array}\right)
$$

and we recover formula (77). Hence, this asymptotic representation is still valid for $r<|z| \leq$ 1 .

Finally, in the domain $\Omega_{0}$,

$$
N(z)=\left(\begin{array}{cc}
0 & D_{\mathrm{i}}(z) / \tau \\
-\tau / D_{\mathrm{i}}(z) & 0
\end{array}\right), \quad K(z)=I, \quad H(z)=I .
$$

Hence,

$$
Y(z)=S(z)\left(\begin{array}{cc}
0 & D_{\mathrm{i}}(z) / \tau \\
-\tau / D_{\mathrm{i}}(z) & 0
\end{array}\right)
$$

and

$$
\Phi_{n}(z)=Y_{11}(z)=-S_{12}(z) \frac{\tau}{D_{\mathrm{i}}(z)}=-\frac{\tau}{D_{\mathrm{i}}(z)}\left(\sum_{k=0}^{\infty} f_{n}^{(2 k+1)}(z)\right), \quad|z|<r .
$$

This concludes the proof of Theorem 1,

Proof of Theorem [2] The asymptotic expansion for the leading coefficient $\kappa_{n}$ is a straightforward consequence of formula (62) and the Riemann-Hilbert steepest descent analysis performed above. Indeed, by (75) and (78),

$$
Y_{21}(z)=-\frac{\tau S_{22}(z)}{D_{\mathrm{i}}(z)}=-\frac{\tau}{D_{\mathrm{i}}(z)}\left(1+\sum_{k=1}^{\infty} g_{n}^{(2 k)}(z)\right)
$$

and the statement follows from (16) and (62).

Proof of Proposition 2. Assume first that $|z| \leq\left(\rho^{\prime}+\rho\right) / 2$. Then $t^{n} \mathcal{F}(t) /(t-z)$ is a meromorphic function of $t$ in the annulus $\left(\rho^{\prime}+\rho\right) / 2<|t|<r$ whose only poles are $a_{1}, \ldots, a_{u}$. Hence, taking $s=\left(3 \rho+\rho^{\prime}\right) / 4$, we may deform the path of integration used in the definition of $f_{n}^{(1)}$ into the one depicted in Figure 5, left, obtaining that

$$
f_{n}^{(1)}(z)=-\frac{1}{2 \pi i \tau^{2}} \oint_{\mathbb{T}_{r}} \frac{\mathcal{F}(t) t^{n}}{t-z} d t=-\frac{1}{\tau^{2}} \sum_{k=1}^{u} \operatorname{res}_{t=a_{k}}\left(\mathcal{F}(t) \frac{t^{n}}{t-z}\right)-\frac{1}{2 \pi i \tau^{2}} \oint_{\mathbb{T}_{s}} \frac{\mathcal{F}(t) t^{n}}{t-z} d t .
$$

Since $|z| \leq\left(\rho^{\prime}+\rho\right) / 2$, we have

$$
\left|\oint_{\mathbb{T}_{\widehat{r}}} \frac{t^{n} \mathcal{F}(t)}{t-z} d t\right| \leq 4 \pi \max _{|t|=\widehat{r}} \mathcal{F}(t) \widehat{r}^{n}
$$



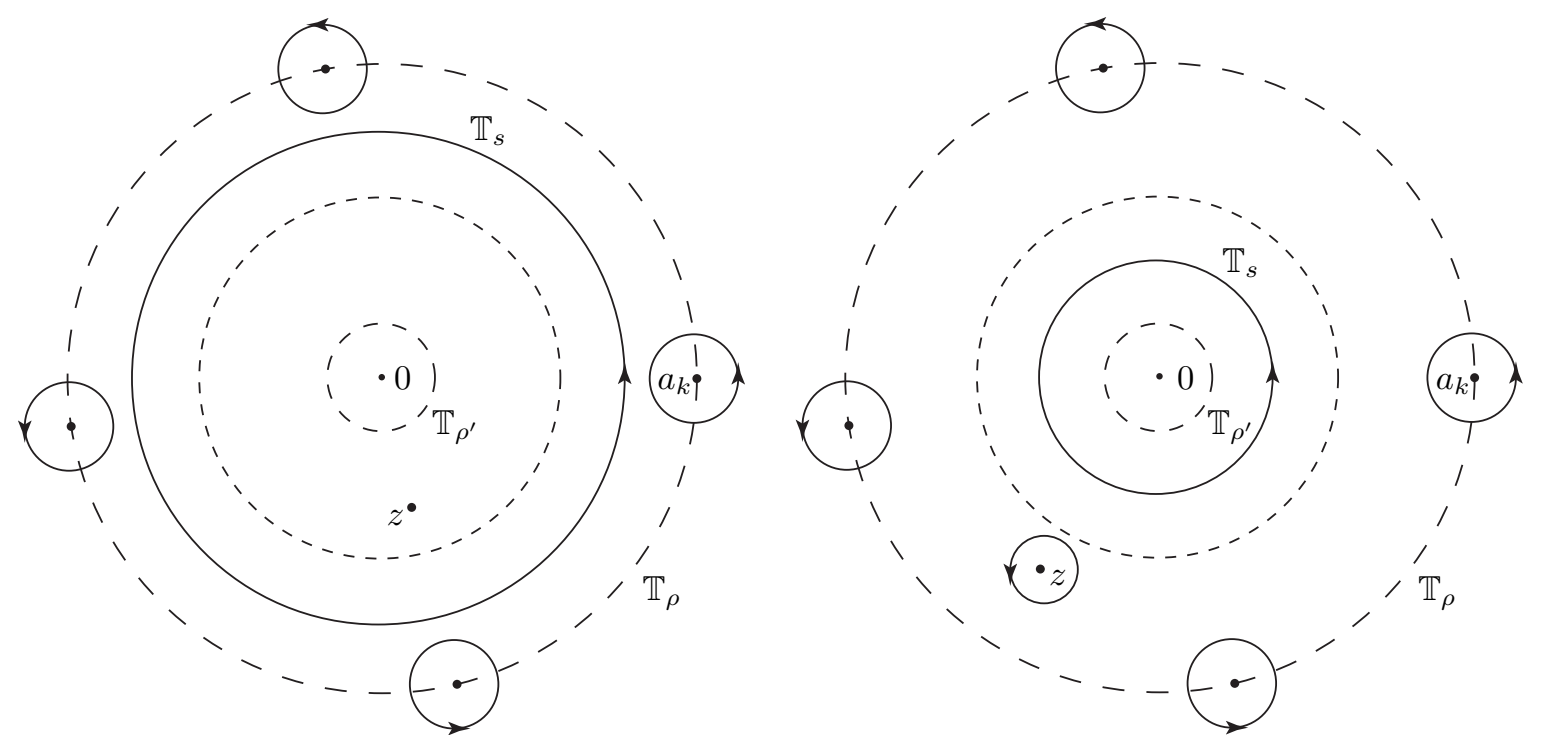

Figure 5: Case of a finite number of singularities on $\mathbb{T}_{\rho}$.

Hence, taking into account (38)-(39) we obtain (42) for $|z| \leq\left(\rho^{\prime}+\rho\right) / 2$, with $\delta=s / \rho$. Obviously, as long as $|z| \leq s$, (41) is a consequence of (42).

Assume now that $\left(\rho^{\prime}+\rho\right) / 2<|z| \leq r^{\prime}$. Then $t^{n} \mathcal{F}(t) /(t-z)$ is a meromorphic function of $t$ in the annulus $\rho^{\prime}<|t|<r$ whose poles are $a_{1}, \ldots, a_{u}$ and $z$. If $z \notin\left\{a_{1}, \ldots, a_{u}\right\}$, the pole of $t^{n} \mathcal{F}(t) /(t-z)$ at $t=z$ is simple, and

$$
\underset{t=z}{\operatorname{res}}\left(\mathcal{F}(t) \frac{t^{n}}{t-z}\right)=z^{n} \mathcal{F}(z) \text {. }
$$

Deforming the path of integration $\mathbb{T}_{r}$ for $f_{n}^{(1)}$ into the one depicted in Figure 5 , right, with $s=\left(3 \rho^{\prime}+\rho\right) / 4$, we obtain that

$$
f_{n}^{(1)}(z)=-\frac{1}{\tau^{2}} z^{n} \mathcal{F}(z)-\frac{1}{\tau^{2}} \sum_{k=1}^{u} \operatorname{res}_{t=a_{k}}\left(\mathcal{F}(t) \frac{t^{n}}{t-z}\right)-\frac{1}{2 \pi i \tau^{2}} \oint_{\mathbb{T}_{s}} \frac{\mathcal{F}(t) t^{n}}{t-z} d t .
$$

However, since $|z|>\left(\rho+\rho^{\prime}\right) / 2$, the same estimate (80) for the last term in this identity remains valid. This proves (41), which remains valid after possible cancellations even for $z \in\left\{a_{1}, \ldots, a_{u}\right\}$. Finally, for any compact set $K \subset \mathbb{D}_{\rho}$, we have that $\max _{z \in K}|z / \rho| \leq \delta<1$, and (42) follows from (41).

Proof of Theorem [3. Observe first that if $a, 0<|a|<1$, is a pole of an analytic function $f$ 
in $|z|<1$, and in a neighborhood of $z=a$ the Laurent expansion of $f$ is

$$
f(z)=\frac{f_{-m}}{(z-a)^{m}}+\frac{f_{-m+1}}{(z-a)^{m-1}}+\ldots, \quad f_{-m} \neq 0, \quad m \in \mathbb{N},
$$

then

$$
f(t) t^{n}=f(t)(t-a+a)^{n}=f(t) \sum_{j=0}^{n}\left(\begin{array}{l}
n \\
j
\end{array}\right) a^{n-j}(t-a)^{j},
$$

and for $n \geq m$ we get

$$
\underset{t=a}{\operatorname{res}}\left(f(t) t^{n}\right)=\sum_{j=0}^{m-1}\left(\begin{array}{l}
n \\
j
\end{array}\right) a^{n-j} f_{-j-1}=f_{-m} a^{n-m+1}\left(\begin{array}{c}
n \\
m-1
\end{array}\right)\left(1+h_{n}\right),
$$

where $h_{n}=0$ for $m=1$, and for $m \geq 2$,

$$
\left|h_{n}\right| \leq \frac{C_{1}}{n} \frac{\max _{0 \leq j \leq m-2}\left|f_{-j-1}\right|}{\left|f_{-m}\right|}
$$

and constant $C_{1}$ depends on $a$ and $m$ only.

Thus, with assumptions of Theorem 3 for $k=1, \ldots, \ell$,

$$
\underset{t=a_{k}}{\operatorname{res}}\left(\mathcal{F}(t) \frac{t^{n}}{t-z}\right)=f_{-m, k}(z) a_{k}^{n-m+1}\left(\begin{array}{c}
n \\
m-1
\end{array}\right)\left(1+h_{n, k}(z)\right),
$$

with

$$
f_{-m, k}(z)=\lim _{z \rightarrow a_{k}} \frac{\mathcal{F}(t)\left(t-a_{k}\right)^{m}}{t-z}=\frac{D_{\mathrm{i}}\left(a_{k}\right) \widehat{D}_{\mathrm{e}}\left(a_{k}\right)}{a_{k}-z} .
$$

Furthermore, for $z \notin B_{\varepsilon}\left(a_{k}\right)$ we have that

$$
\left|h_{n, k}(z)\right| \leq \frac{C_{2}}{n} \frac{1}{\left|z-a_{k}\right|^{m-1}} \leq \frac{C_{2}}{n} \frac{1}{\varepsilon^{m-1}},
$$

and constant $C_{2}$ depends on $\rho, r, \Lambda$ and $m$, but neither on $z$ nor $n$.

The multiplicity $m^{\prime}$ of a non-dominant pole $a_{k}, k>\ell$, is strictly less than $m$; by (81) we obtain again that as long as $z \notin B_{\varepsilon}\left(a_{k}\right)$,

$$
\underset{t=a_{k}}{\operatorname{res}}\left(\mathcal{F}(t) \frac{t^{n}}{t-z}\right)=\mathcal{O}\left(\rho^{n} n^{m^{\prime}-1}\right), \quad m^{\prime}<m,
$$

uniformly in $z$. 
Assumptions of Theorem 3 yield those of Proposition 2. Hence, plugging formulas (82)(84) into (41)-(42), we obtain (43)-(44). Estimate (45) is a straightforward consequence of (43) since we may assume now $r^{3}<\rho<r$.

For the last statement it is sufficient to observe that given a compact subset $K$ in $|z|<\rho$, by (45),

$$
\frac{1}{a_{1}^{n-m+1}}\left(\begin{array}{c}
n \\
m-1
\end{array}\right)^{-1} \Phi_{n}(z)
$$

is a normal family in $|z|<\rho$, and every limit of a convergent subsequence is a rational function with at most $\ell-1$ zeros on $K$.

Proof of Theorem 4. We carry out the proof in the spirit of [Sim04b], but with an additional difficulty generated by the "floating" zeros of $G_{n}$.

Let $\varepsilon>0$; without loss of generality we may assume that $a_{1}=\rho>0$. Then for $z \in \mathcal{B}(\varepsilon)$ we may rewrite (43) using the notation (48):

$$
\frac{\tau}{D_{\mathrm{e}}(z)} \rho^{-n}\left(\begin{array}{c}
n \\
m-1
\end{array}\right)^{-1} \Phi_{n}(z)=\left(\begin{array}{c}
n \\
m-1
\end{array}\right)^{-1}\left(\frac{z}{\rho}\right)^{n}+\frac{G_{n}(z)}{\rho^{m-1} \mathcal{F}(z)}+H_{n}(z),
$$

where

$$
H_{n}(z)=\frac{\tau}{D_{\mathrm{e}}(z)} \rho^{-n}\left(\begin{array}{c}
n \\
m-1
\end{array}\right)^{-1} h_{n}(z)
$$

According to (44), there exists a constant $C=C(\varepsilon)$ such that

$$
\left|H_{n}(z)\right| \leq \frac{C}{n}, \quad z \in \mathcal{B}(\varepsilon)
$$

Furthermore, $\left\{G_{n}\right\}$ is an equicontinuous family of rational functions of a bounded degree. In consequence, there exist constants $q=q(\varepsilon)$ and $Q=Q(\varepsilon)$ such that for all $n \in \mathbb{N}$,

$$
z \in \mathcal{B}(\varepsilon) \backslash \mathcal{Z}_{\varepsilon}\left(G_{n}\right) \quad \Rightarrow \quad 0<q \leq\left|\frac{G_{n}(z)}{\rho^{m-1} \mathcal{F}(z)}\right| \leq Q<+\infty
$$

and a constant $\delta=\delta(\varepsilon)>0$ such that

$$
z, \zeta \in \mathcal{B}(\varepsilon) \backslash \mathcal{Z}_{\varepsilon}\left(G_{n}\right),|z-\zeta|<\delta \Rightarrow\left|\frac{G_{n}(z)}{\rho^{m-1} \mathcal{F}(z)}-\frac{G_{n}(\zeta)}{\rho^{m-1} \mathcal{F}(\zeta)}\right| \leq \frac{q}{4}
$$

For $v_{n}$ defined in (50), there exists $n_{0} \in \mathbb{N}$ such that for $n \geq n_{0}$,

$$
v_{n} \leq r-\varepsilon, \quad \frac{C}{n} \leq \frac{q}{4}, \quad \text { and } \quad(1-\delta / 2)^{n}<\frac{q}{2}<\frac{3 Q}{2}<(1+\delta / 2)^{n} .
$$


Fix $n \geq n_{0}$ and assume that $\zeta,|\zeta|=v_{n}$, is such that $B_{\delta}(\zeta) \subset \mathcal{B}(\varepsilon) \backslash \mathcal{Z}_{\varepsilon}\left(G_{n}\right)$. Denote

$$
\frac{G_{n}(\zeta)}{\rho^{m-1} \mathcal{F}(\zeta)}=s e^{i \alpha} \quad \text { and } \quad \Delta_{n}(z) \stackrel{\text { def }}{=} \frac{G_{n}(z)}{\rho^{m-1} \mathcal{F}(z)}-s e^{i \alpha}
$$

Choose a neighborhood of $t=\zeta$ of the following form:

$$
\begin{aligned}
I_{n} & =I_{n}\left(k_{1}, k_{2}\right) \\
& \stackrel{\text { def }}{=}\left\{z \in \mathbb{C}: v_{n}(1-\delta / 2)<|z|<v_{n}(1+\delta / 2), \frac{\alpha+2 k_{1} \pi}{n}<\arg (z)<\frac{\alpha+2 k_{2} \pi}{n}\right\},
\end{aligned}
$$

where integers $k_{1} \leq k_{2}$ satisfy $\zeta \in I_{n}$ and $k_{2}-k_{1} \leq \delta n / 8$. Then $I_{n}\left(k_{1}, k_{2}\right) \subset \mathcal{B}(\varepsilon) \backslash \mathcal{Z}_{\varepsilon}\left(G_{n}\right)$. By (87), $\left|\Delta_{n}(z)\right|<s / 4$ on the boundary $\partial I_{n}$. In consequence,

$$
z \in \partial I_{n} \quad \Rightarrow \quad\left|\Delta_{n}(z)+H_{n}(z)\right| \leq\left|\Delta_{n}(z)\right|+\left|H_{n}(z)\right|<\frac{s}{2},
$$

where we have taken into account (86) and (88).

With the notation introduced above we rewrite (85) as

$$
\frac{\tau}{D_{\mathrm{e}}(z)} \rho^{-n}\left(\begin{array}{c}
n \\
m-1
\end{array}\right)^{-1} \Phi_{n}(z)=b_{n}(z)+\Delta_{n}(z)+H_{n}(z)
$$

where

$$
b_{n}(z)=\left(\begin{array}{c}
n \\
m-1
\end{array}\right)^{-1}\left(\frac{z}{\rho}\right)^{n}+s e^{i \alpha}
$$

For $|z|=v_{n}(1-\delta / 2)$

$$
\left|b_{n}(z)\right| \geq s-(1-\delta / 2)^{n}>s / 2,
$$

where we have taken into account a condition in (88). Analogously, when $|z|=v_{n}(1+\delta / 2)$,

$$
\left|b_{n}(z)\right| \geq(1+\delta / 2)^{n}-s>s / 2,
$$

again by (88). Finally, when $\arg (z)=(\alpha+2 k \pi) / n, k \in \mathbb{Z}$,

$$
\arg \left(\left(\begin{array}{c}
n \\
m-1
\end{array}\right)^{-1}\left(\frac{z}{\rho}\right)^{n}\right)=\alpha+2 k \pi \Rightarrow\left|b_{n}(z)\right|=\left(\begin{array}{c}
n \\
m-1
\end{array}\right)^{-1}\left(\frac{|z|}{\rho}\right)^{n}+s>s .
$$

In other words, we have that $\left|b_{n}(z)\right|>s / 2$ on $z \in \partial I_{n}$. Taking into account (89), by Rouche's theorem, $b_{n}$ and

$$
\frac{\tau}{D_{\mathrm{e}}(z)} \rho^{-n}\left(\begin{array}{c}
n \\
m-1
\end{array}\right)^{-1} \Phi_{n}(z)
$$


have the same number of zeros in $I_{n}\left(k_{1}, k_{2}\right)$. But every pie slice $I_{n}(k, k+1)$ of angle $2 \pi / n$ contains only one zero of $b_{n}$, so in general $\Phi_{n}$ has exactly $k_{2}-k_{1}$ zeros in every pie slice $I_{n}\left(k_{1}, k_{2}\right) \subset \mathcal{B}(\varepsilon) \backslash \mathcal{Z}_{\varepsilon}\left(G_{n}\right)$ of angle $2 \pi\left(k_{2}-k_{1}\right) / n$.

On the other hand, if $z_{0}$ is a zero of $\Phi_{n}$, by (90), $b_{n}\left(z_{0}\right)=-\Delta_{n}\left(z_{0}\right)-H_{n}\left(z_{0}\right)$, or

$$
\left(\begin{array}{c}
n \\
m-1
\end{array}\right)^{-1}\left(\frac{z_{0}}{\rho}\right)^{n}=-s e^{i \alpha}-\Delta_{n}\left(z_{0}\right)-H_{n}\left(z_{0}\right)
$$

so that

$$
\left(\begin{array}{c}
n \\
m-1
\end{array}\right)^{-1}\left(\frac{\left|z_{0}\right|}{\rho}\right)^{n}=s+\mathcal{O}\left(\frac{1}{n}\right) \Rightarrow\left|z_{0}\right|=\rho\left(1+\frac{1}{n} \log \left(\begin{array}{c}
n \\
m-1
\end{array}\right)+\mathcal{O}\left(\frac{1}{n}\right)\right) .
$$

Analogously, if $z_{0}, z_{1}$ are the zeros of $\Phi_{n}$ in two consecutive pie slices, $I_{n}(k-1, k)$ and $I_{n}(k, k+1)$, then

$$
\arg \left(z_{1}\right)-\arg \left(z_{0}\right)=\frac{2 \pi}{n}+\mathcal{O}\left(\frac{1}{n^{2}}\right)
$$

Proof of Proposition [3. By (45),

$$
Z \subset \bigcap_{k \geq 1} \overline{\bigcup_{n \geq k} \mathcal{Z}\left(G_{n}\right)}
$$

with $G_{n}$ defined in (48), and it is sufficient to describe all the possible limit points of $\left\{G_{n}\right\}$. Observe that with the notation introduced before,

$$
\begin{aligned}
G_{n}(z) & =\sum_{k=1}^{\ell} \frac{D_{\mathrm{i}}\left(a_{k}\right) \widehat{D}_{\mathrm{e}}\left(a_{k}\right)}{a_{k}-z} \exp \left(2 \pi i(n-m+1) \theta_{k}\right) \\
& =\sum_{k=1}^{\ell} \frac{D_{\mathrm{i}}\left(a_{k}\right) \widehat{D}_{\mathrm{e}}\left(a_{k}\right)}{a_{k}-z} \exp \left(2 \pi i \sum_{j=1}^{v} r_{k j}(n-m+1) \theta_{j}\right) .
\end{aligned}
$$

The case $v=1$ is trivial; assume $v \geq 2$. By Kronecker's theorem (also known as Kronecker-Weyl theorem, see e.g. Cass57, Ch. III]), since $\theta_{1}=1, \theta_{2}, \ldots, \theta_{v}$ are rationally independent then for any real numbers $X_{2}, \ldots, X_{v}$ there exists a sub-sequence $\left\{n_{j}\right\} \subset \mathbb{N}$ such that

$$
\lim _{n_{j}} e^{2 \pi i\left(\left(n_{j}-m+1\right) \theta_{k}-\beta_{k}\right)}=1, \quad k=2, \ldots, v,
$$

and $\left(n_{j}-m+1\right) p_{k} / q_{k}, k=1, \ldots, \ell$, have limits modulo $\mathbb{Z}$. This shows that the set of limit points of $\left\{G_{n}\right\}$ is parameterized by $0 \leq s_{k}<q_{k}, s_{k} \in \mathbb{Z}, k=1, \ldots, \ell$ and $X_{2}, \ldots, X_{v} \in \mathbb{R}$, and is given in the left hand side of (551). Now the statement follows for $v \geq 2$. 


\section{Some examples}

We illustrate our results with some examples. Estimates (38)-(39) justify concentrating on the asymptotics of $f_{n}^{(1)}$ and of its zeros in $\mathbb{D}_{r}$.

Consider first the simplest case of a polynomial weight inducing a single pole of $D_{\mathrm{e}}$ inside $\mathbb{T}_{1}$. Let $w(z)=|z-a|^{2},|z|=1$, where without loss of generality we may assume $a>0, a \neq 1$ (the case $a=0$ is trivial). Then the normalized representation of $w$, according to Definition 2] is

$$
w(z)=\left|\frac{z-a}{z}\right|^{2}, \quad \text { if } a<1, \quad \text { and } \quad w(z)=|1-a z|^{2}, \quad \text { if } a>1 .
$$

Assume $0<a<1$; in this case

$$
D_{\mathrm{i}}(z)=1-a z, \quad D_{\mathrm{e}}(z)=\frac{z}{z-a}, \quad \tau=1, \quad \text { and } \quad \mathcal{F}(z)=\frac{z(1-a z)}{z-a} .
$$

In consequence, with $a<r<1$,

$$
f_{n}^{(1)}(z)= \begin{cases}\frac{z^{n+1}(1-a z)-a^{n+1}\left(1-a^{2}\right)}{a^{n+1} \frac{1-a^{2}}{z-a},}, & \text { if }|z|<r \\ \text { if }|z|>r .\end{cases}
$$

Observe that for $|z|<r$ this is a polynomials of degree $n$, such that $f_{n}^{(1)}(a) \neq 0$, and whose zeros distribute asymptotically uniformly on $\mathbb{T}_{|a|}$, leaving a gap at $z=a$.

Furthermore,

$$
f_{n}^{(2)}(z)=a^{2 n}\left(1-a^{2}\right)(1-a z), \quad z \in \mathbb{D}_{1 / r},
$$

and in consequence,

$$
f_{n}^{(3)}(z)=a^{2 n}\left(1-a^{2}\right) \frac{z^{n+1}(1-a z)^{2}-a^{n+1}\left(1-a^{2}\right)^{2}}{a-z}, \quad z \in \mathbb{D}_{r} .
$$

Then by (32),

$$
\begin{aligned}
\Phi_{n}(z)= & -\frac{1}{1-a z}\left(\frac{z^{n+1}(1-a z)-a^{n+1}\left(1-a^{2}\right)}{a-z}\right. \\
& \left.+a^{2 n}\left(1-a^{2}\right) \frac{z^{n+1}(1-a z)^{2}-a^{n+1}\left(1-a^{2}\right)^{2}}{a-z}+\mathcal{O}\left(a^{5 n}\right)\right),
\end{aligned}
$$

which is valid locally uniformly in $\mathbb{D}_{|a|+\varepsilon}$, with $\varepsilon>0$. Obviously, we can get further terms in this asymptotic expansion.

Analogously,

$$
g_{n}^{(1)}(z)=a^{n} \frac{1-a^{2}}{1-a z}, \quad z \in \mathbb{D}_{1 / r}
$$


and

$$
g_{n}^{(2)}(z)=-a^{n}\left(1-a^{2}\right) \frac{z^{n+1}-a^{n+1}}{z-a}, \quad z \in \mathbb{D}_{r} .
$$

Hence, using Theorem [2] we have the following asymptotics for the leading coefficient $\kappa_{n}$ of orthonormal polynomials $\varphi_{n}$ :

$$
\kappa_{n}^{2}=\frac{1}{2 \pi}\left(1-a^{2 n}\left(1-a^{2}\right)+\mathcal{O}\left(r^{4 n}\right)\right), \quad n \rightarrow \infty .
$$

Much richer is the case of a polynomial weight inducing two dominant poles of $D_{\mathrm{e}}$ inside $\mathbb{T}_{1}$. Consider

$$
w(z)=|(z-a)(z-b)|^{2}, \quad 0<a=|b|<1 .
$$

Then

$$
D_{\mathrm{i}}(z)=(1-a z)(1-\bar{b} z), \quad D_{\mathrm{e}}(z)=\frac{z^{2}}{(z-a)(z-b)}, \quad \tau=1
$$

and

$$
\mathcal{F}(z)=z^{2} \frac{(1-a z)(1-\bar{b} z)}{(z-a)(z-b)} .
$$

Let us denote $b=a e^{i \pi \theta}$, and fix $a<r<1$. Then we have for $z \in \mathbb{D}_{r}$,

$$
f_{n}^{(1)}(z)=-a^{n}\left\{\mathcal{F}(z)\left(\frac{z}{a}\right)^{n}+a \frac{1-a^{2}}{1-e^{i \pi \theta}}\left[\frac{1-a^{2} e^{-i \pi \theta}}{a-z}-e^{i \pi(n+2) \theta} \frac{1-a^{2} e^{i \pi \theta}}{b-z}\right]\right\} .
$$

Hence, for $|z|<|a|$, the zeros of $f_{n}^{(1)}$ are asymptotically close to the solutions of

$$
\frac{1-a^{2} e^{-i \pi \theta}}{a-z}=e^{i \pi(n+2) \theta} \frac{1-a^{2} e^{i \pi \theta}}{b-z}
$$

or in other words, close to

$$
z=a e^{i \pi \theta} \frac{e^{i \pi(n+1) \theta}-A}{e^{i \pi(n+2) \theta}-A}, \quad \text { where } \quad A \stackrel{\text { def }}{=} \frac{1-a^{2} e^{-i \pi \theta}}{1-a^{2} e^{i \pi \theta}}=\frac{\overline{1-a^{2} e^{i \pi \theta}}}{1-a^{2} e^{i \pi \theta}}, \quad|A|=1 .
$$

Let us consider some particular cases. First, $\theta=\sqrt{2} \notin \mathbb{Q}$ illustrates Remark 14. In this case $Z \cap \mathbb{D}_{|a|}$ is parameterized by

$$
z=a \frac{e^{i \pi \alpha}-A}{e^{i \pi \alpha}-e^{-i \pi \theta} A}, \quad \alpha \in \mathbb{R},
$$

which is a straight line passing through the origin (cf. the numerical example in Fig. 2, left). The rest of the zeros tends to the circle $\mathbb{T}_{1 / 2}$ exhibiting the equidistribution pattern predicted by Theorem 4 (see Fig. 6). 

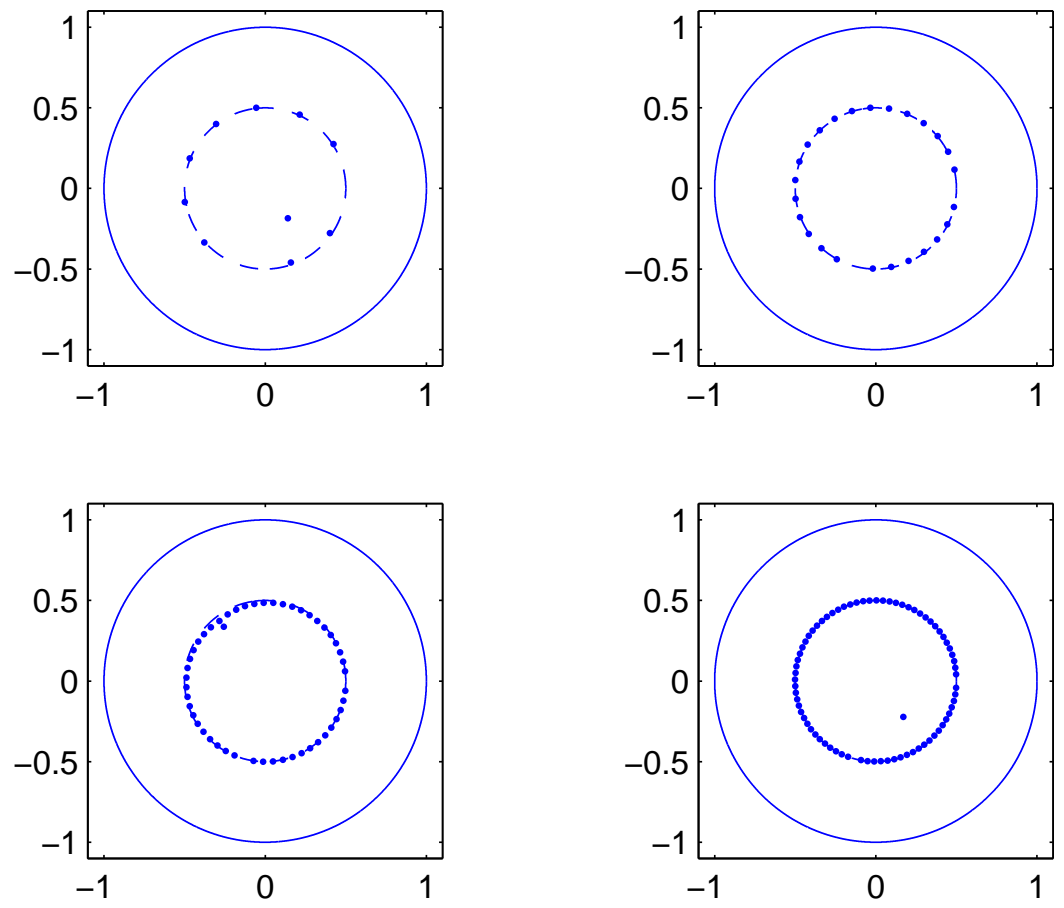

Figure 6: Zeros of $\Phi_{n}$ for $n=10,25,50,75$ (from left to right and from top to bottom) with $w(z)=\left|(z-1 / 2)\left(z-\zeta_{1} / 2\right)\right|^{2}, z \in \mathbb{T}_{1}$, where $\zeta_{1}=\exp (\pi i \sqrt{2})$. The dashed line is the circle of radius 0.5 .

Next, if $\theta=1 / 2(b=i a)$, we have by Proposition 3 that the zero accumulation set $Z \cap D_{|a|}$ is finite. Namely, points (92) in this case have the following form:

$$
\begin{aligned}
& n=4 k, k \in \mathbb{N}, \Rightarrow \quad z_{0}=a i \frac{i-A}{-1-A} \in \mathbb{D}_{|a|} \text { if } \arg (A) \in(-\pi / 4,3 \pi / 4) \quad \bmod 2 \pi, \\
& n=4 k+1, k \in \mathbb{N}, \quad \Rightarrow \quad z_{1}=a i \frac{-1-A}{-i-A} \in \mathbb{D}_{|a|} \text { if } \arg (A) \in(\pi / 4,5 \pi / 4) \quad \bmod 2 \pi, \\
& n=4 k+2, k \in \mathbb{N}, \quad \Rightarrow \quad z_{2}=a i \frac{-i-A}{1-A} \in \mathbb{D}_{|a|} \text { if } \arg (A) \in(3 \pi / 4,7 \pi / 4) \quad \bmod 2 \pi, \\
& n=4 k+3, k \in \mathbb{N}, \quad \Rightarrow \quad z_{3}=a i \frac{1-A}{i-A} \in \mathbb{D}_{|a|} \text { if } \arg (A) \in(5 \pi / 4,9 \pi / 4) \quad \bmod 2 \pi .
\end{aligned}
$$

Hence, $Z \cap \mathbb{D}_{|a|}$ has at most 2 points, and unless $\theta= \pm \pi / 4 \bmod \pi$, the sequence $\left\{f_{n}^{(1)}\right\}$ 

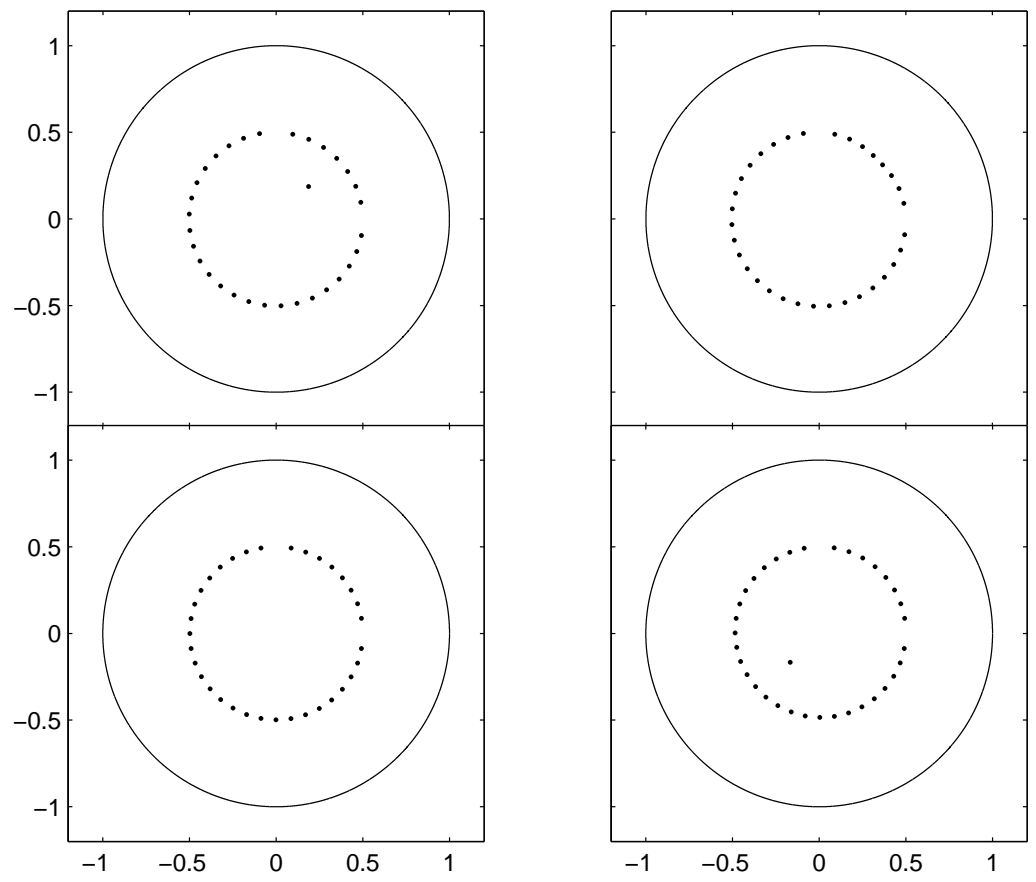

Figure 7: Zeros of $\Phi_{n}$ for $w(z)=|(z-1 / 2)(z-i / 2)|^{2}, z \in \mathbb{T}_{1}$, and $n=32, \ldots, 35$ (from left to right and from top to bottom).

will have zeros in $\mathbb{D}_{|a|}$ for two consecutive indices $n$, and has no zeros inside $\mathbb{T}_{|a|}$ for the next two. For instance, with $a=1 / 2, \arg (A) \approx 0.156 \pi$, and zeros of $\left\{f_{n}^{(1)}\right\}$ (and also zeros of $\left.\left\{\Phi_{n}\right\}\right)$ have limits in $\mathbb{D}_{|a|}$ only for $n=4 k$ and $n=4 k+3, k \in \mathbb{N}$, which are

$$
s_{1}=\frac{3}{16}(1+i), \quad s_{2}=-\frac{1}{6}(1+i),
$$

(compare with the numerical experiment in Fig. 17).

On the other hand, by (38)-(39) and by (91),

$$
\frac{\Phi_{n}(0)}{\Phi_{n-1}(0)} \sim \frac{f_{n}^{(1)}(0)}{f_{n-1}^{(1)}(0)}=a \frac{e^{i \pi(n+2) \theta}-A}{e^{i \pi(n+1) \theta}-A}\left(1+\mathcal{O}\left(a^{2 n}\right)\right), \quad n \rightarrow \infty .
$$

Let us compare it with the results of [BRLLS01] for $\theta=1 / 2$. Denoting

$$
b_{j} \stackrel{\text { def }}{=} \lim _{n=j} \frac{\Phi_{n}(0)}{\bmod 4}, \quad j=1, \ldots, 4,
$$


we have by (94),

$$
b_{1}=a \frac{-i-A}{-1-A}, \quad b_{2}=a \frac{1-A}{-i-A}, \quad b_{3}=a \frac{i-A}{1-A}, \quad b_{4}=a \frac{-1-A}{i-A} .
$$

Observe that $\left|b_{1} \ldots b_{4}\right|^{1 / 4}=|a|$. Using the notation of [BRLLS01], we compute

$$
\Delta_{3}^{(j)}(z)=\left|\begin{array}{ccc}
z+b_{j} & a b_{j+1} & 0 \\
1 & a+b_{j+1} & z b_{j+2} \\
0 & 1 & z+b_{j+2}
\end{array}\right|, \quad j=1, \ldots, 4,
$$

(where we take $b_{5}=b_{1}$ and $b_{6}=b_{2}$ ), and take

$$
\Delta=\bigcup_{j=1}^{4}\left\{z: \Delta_{3}^{(j)}(z)=0\right\} .
$$

For $a=1 / 2$ it is straightforward to compute that $\Delta \cap \mathbb{D}_{|a|}=\left\{s_{1}, s_{2}\right\}$, where $s_{1}, s_{2}$ are defined in (93). In other words, this case gives an example when the whole set $\Delta \cap \mathbb{D}_{|a|}$ gives the accumulation points of zeros of $\left\{\Phi_{n}\right\}$ (cf. Remark 1 in BRLLS01]).

Finally, we dwell very briefly on the case when $D_{\mathrm{e}}$ has an essential singularity on $\mathbb{T}_{\rho}$ analyzing two examples.

Let first the weight function be given by

$$
w(t)=\left|\exp \left(\frac{1}{a-t}\right)\right|^{2}, \quad t \in \mathbb{T}_{1},
$$

with $a \in \mathbb{D}_{1}$. Without loss of generality we may assume $0<a<1$, so that $\rho=a$. Then

$$
D_{\mathrm{i}}(t)=\exp \left(\frac{t}{a t-1}\right), \quad D_{\mathrm{e}}(t)=\exp \left(\frac{1}{t-a}\right), \quad \tau=D_{\mathrm{e}}(\infty)=1,
$$

and

$$
\mathcal{F}(t)=\exp \left(\frac{t}{a t-1}+\frac{1}{t-a}\right) .
$$

Again wee have to analyze the asymptotic behavior of the sequence of Cauchy transforms

$$
f_{n}^{(1)}(z)=-\frac{1}{2 \pi i} \oint_{\mathbb{T}_{r}} \frac{t^{n} \mathcal{F}(t)}{t-z} d t=-\frac{1}{2 \pi i} \oint_{\mathbb{T}_{r}} \frac{e^{n \Psi_{n}(t)}}{t-z} d t, \quad z \in \mathbb{D}_{r},
$$

where we have used the notation

$$
\Psi_{n}(t) \stackrel{\text { def }}{=} \log t+\frac{1}{n} \log \mathcal{F}(z)=\log t+\frac{1}{n}\left(\frac{t}{a t-1}+\frac{1}{t-a}\right) .
$$


Observe that we cannot apply the classical steepest descent method to the last integral in (96), since $\Psi_{n}$ depends on $n$.

Let $\gamma_{n}$ be a positively oriented closed Jordan curve encircling $a$ and contained within $\mathbb{D}_{r}$, and eventually depending on $n$. Define

$$
I_{n}(z) \stackrel{\text { def }}{=}-\frac{1}{2 \pi i} \oint_{\gamma_{n}} \frac{e^{n \Psi_{n}(t)}}{t-z} d t
$$

Then

$$
f_{n}^{(1)}(z)= \begin{cases}-z^{n} \mathcal{F}(z)+I_{n}(z), & \text { if } z \text { is in } \mathbb{D}_{r} \text { and outside } \gamma_{n} \\ I_{n}(z), & \text { if } z \text { is inside } \gamma_{n} .\end{cases}
$$

For all sufficiently large $n$, function $\Psi_{n}$ has four simple saddle points, $t_{ \pm}$and $1 / t_{ \pm}$,

$$
0<t_{-}<a<t_{+}<1<1 / t_{+}<1 / a<1 / t_{-},
$$

such that

$$
t_{ \pm}=a \pm \frac{\sqrt{a}}{\sqrt{n}}+\mathcal{O}(1 / n), \quad n \rightarrow \infty
$$

Function $\Psi_{n}$ is meromorphic in the cut disc $\mathbb{D}_{1} \backslash(-1,0]$, and its level curves $\Gamma=\{t \in$ $\left.\mathbb{C} \backslash(-\infty, 0]: \operatorname{Im} \Psi_{n}(t)=\operatorname{Im} \Psi_{n}\left(t_{+}\right)=0\right\}$ are trajectories of the quadratic differential $\Psi_{n}^{\prime}(t) d t^{2}$ which has a double pole at $t=0$ with real leading coefficient, a pole of order 4 at $a$, and double zeros at $t_{ \pm}$. It is easy to see also that $(0,1) \backslash\{a\} \subset \Gamma$. The typical local structure of the level set $\Gamma$ at $t=t_{+}$is in Fig. 8 there are four arcs of $\Gamma$ emanating from $t_{+}$with equal angles; two of these are $(0, a)$ and $(a, 1)$, another two should be vertical arcs passing through $t_{ \pm}$and ending at $(0,1)$. Since an analogous conclusion is valid in a neighborhood of $t_{-}$, we see that necessarily $\Gamma$ contains a closed Jordan loop passing through $t_{ \pm}$, that we choose as $\gamma_{n}$ in (97); see Fig. 9] right. Analogous arguments allow to determine the orthogonal trajectories $\Gamma^{\perp}=\left\{t \in \mathbb{C} \backslash(-\infty, 0]: \operatorname{Re} \Psi_{n}(t)=\operatorname{Re} \Psi_{n}\left(t_{+}\right)\right\}$, see Fig. 9, left. Since $\Psi_{n}\left(t_{-}\right)<\Psi_{n}\left(t_{+}\right)$, we have that $\operatorname{Re} \Psi_{n}(t)<\operatorname{Re} \Psi_{n}\left(t_{+}\right)$in the whole connected component of the complement of $\Gamma^{\perp}$ containing $t_{-}$. In particular, $\operatorname{Re} \Psi_{n}(t)$ attains its strict maximum on $\gamma_{n}$ at $t_{+}$, so this is really the steepest descent curve for $I_{n}$.

Let us take $\delta>0$ and denote

$$
B_{n} \stackrel{\text { def }}{=} B_{\delta / \sqrt{n}}\left(t_{+}\right)=\left\{t \in \mathbb{C}:\left|t-t_{+}\right|<\delta / \sqrt{n}\right\}, \quad \beta_{n}=\partial B_{n}=\left\{t \in \mathbb{C}:\left|t-t_{+}\right|=\delta / \sqrt{n}\right\} .
$$

We assume $n$ large enough, so that $B_{n} \subset \mathbb{D}_{r} \backslash(-\infty, a]$. Observe that $t_{+}$is the only zero of $\Psi_{n}(t)-\Psi_{n}\left(t_{+}\right)$in $B_{n}$, and it is double: in a neighborhood of $t=t_{+}$,

$$
\Psi_{n}(t)-\Psi_{n}\left(t_{+}\right)=\frac{\Psi^{\prime \prime}\left(t_{+}\right)}{2}\left(t-t_{+}\right)^{2}\left(1+\mathcal{O}\left(t-t_{+}\right)\right), \quad t \rightarrow t_{+} .
$$




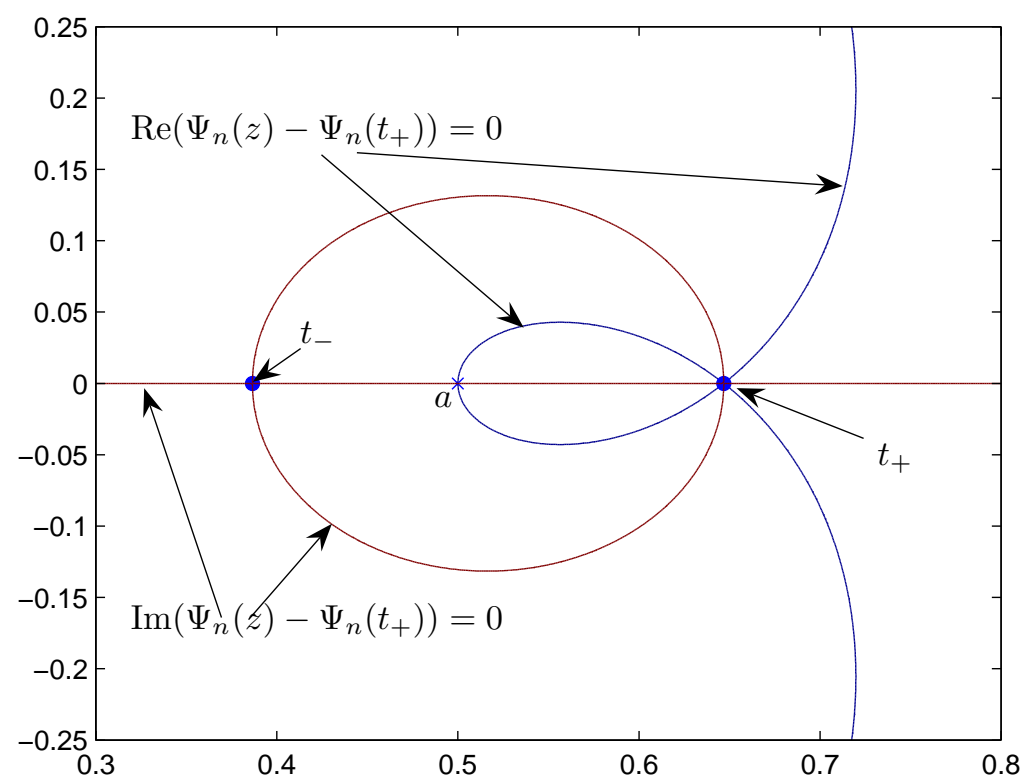

Figure 8: Level curves $\operatorname{Re}\left(\Psi_{n}(z)-\Psi_{n}\left(t_{+}\right)\right)=0$ and $\operatorname{Im}\left(\Psi_{n}(z)-\Psi_{n}\left(t_{+}\right)\right)=0$ in a neighborhood of $t_{ \pm}($for $a=1 / 2$ and $n=30)$.

By (16), $\Psi_{n}^{\prime \prime}\left(t_{+}\right)=2 n^{1 / 2} a^{-3 / 2}(1+o(1))$. Condition $\sqrt{\Psi_{n}(t)-\Psi_{n}\left(t_{+}\right)}>0$ for $t>t_{+}$fixes a single-valued branch of $\sqrt{\Psi_{n}(t)-\Psi_{n}\left(t_{+}\right)}$in $B_{n}$. With this convention, we define

$$
\zeta_{n}(t) \stackrel{\text { def }}{=}-i n^{1 / 4} \sqrt{\Psi_{n}(t)-\Psi_{n}\left(t_{+}\right)},
$$

(where we take the positive square root). It is a conformal mapping of $B_{n}$ such that that $\zeta_{n}\left(\mathbb{R} \cap B_{n}\right) \subset i \mathbb{R}$, and $\zeta_{n}\left(\gamma_{n} \cap B_{n}\right)=\left(-d_{n}, d_{n}\right) \subset \mathbb{R}$; moreover, the arc of $\gamma_{n} \cap B_{n}$ in the upper half plane $\mathbb{C}^{+}$is mapped onto the positive semiaxis. Also

$$
\zeta_{n}(t)=-i \frac{n^{1 / 2}}{a^{3 / 4}}\left(t-t_{+}\right)\left(1+\mathcal{O}\left(t-t_{+}\right)\right), \quad t \rightarrow t_{+},
$$

and for sufficiently large $n$,

$$
\zeta_{n}(t)^{2} \geq \frac{\delta^{2}}{2 a^{3 / 2}}, \quad t \in \gamma_{n} \backslash B_{n}
$$



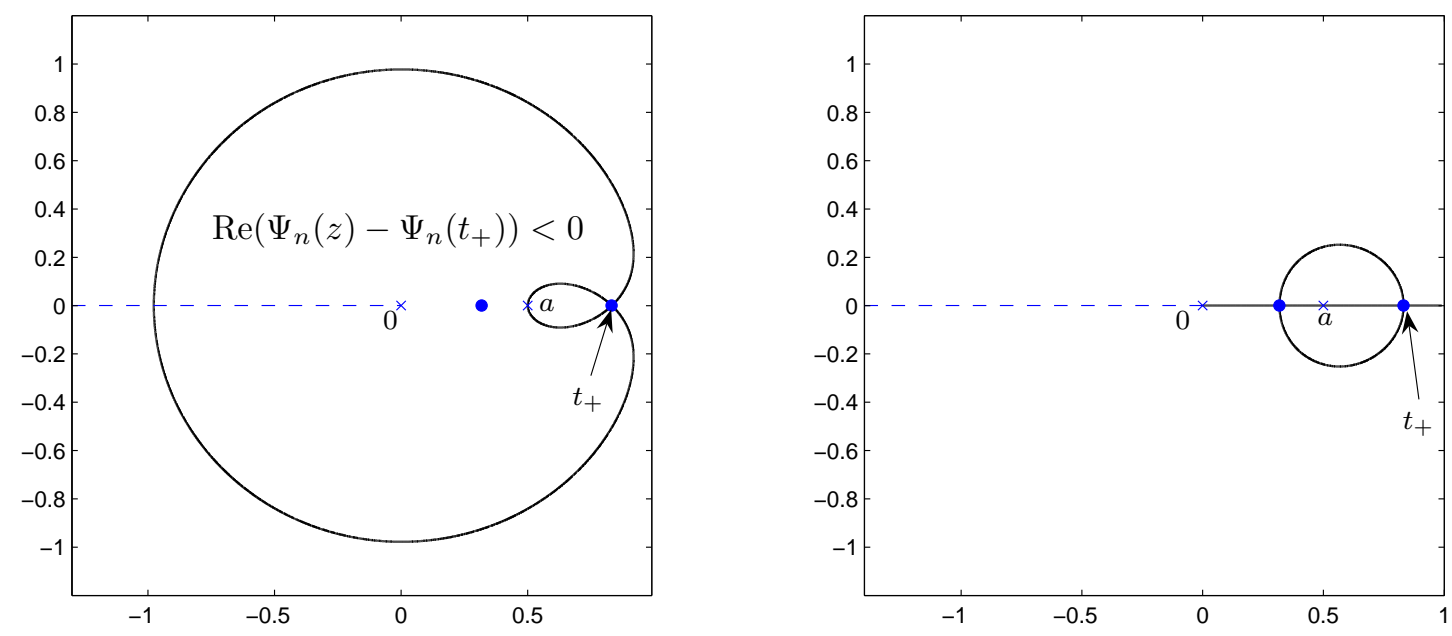

Figure 9: Global structure of the level curves $\operatorname{Re}\left(\Psi_{n}(z)-\Psi_{n}\left(t_{+}\right)\right)=0$ (left) and $\operatorname{Im}\left(\Psi_{n}(z)-\right.$ $\left.\Psi_{n}\left(t_{+}\right)\right)=0$ for $a=1 / 2$ and $n=30$.

For the error function

$$
\begin{aligned}
P_{n}(u) & \stackrel{\text { def }}{=}-e^{n \Psi_{n}\left(t_{+}\right)} \frac{1}{2 \pi i} \int_{-\infty}^{+\infty} \frac{e^{-\sqrt{n} t^{2}}}{t-u} d t \\
& =-\frac{1}{2} e^{n \Psi_{n}\left(t_{+}\right)-\sqrt{n} u^{2}} \operatorname{erfc}\left(-i n^{1 / 4} u\right), \quad u \notin \mathbb{R},
\end{aligned}
$$

we define $F_{n}(z) \stackrel{\text { def }}{=} P_{n}\left(\zeta_{n}(z)\right), z \in B_{n} \backslash \gamma_{n}$. Then $F_{n}$ has the following jump across $\gamma_{n} \cap B_{n}$ :

$$
F_{n,+}(z)-F_{n,-}(z)=-e^{n \Psi_{n}\left(t_{+}\right)} e^{-\sqrt{n} \zeta_{n}(z)^{2}}=-e^{n \Psi_{n}(z)},
$$

matching the jump of $I_{n}$ across the same arc. This fact along with Sokhotsky's formulas show that

$$
I_{n}(z)= \begin{cases}I_{n}^{*}(z)+F_{n}(z)-\frac{1}{2 \pi i} \int_{\beta_{n}} \frac{F_{n}(t)}{t-z} d t, & \text { for } z \in B_{n} \backslash \gamma_{n}, \\ I_{n}^{*}(z)-\frac{1}{2 \pi i} \int_{\beta_{n}} \frac{F_{n}(t)}{t-z} d t, & \text { for } z \in \mathbb{C} \backslash\left(\overline{B_{n}} \cup \gamma_{n}^{*}\right),\end{cases}
$$

where

$$
I_{n}^{*}(z) \stackrel{\text { def }}{=}-\frac{1}{2 \pi i} \int_{\gamma_{n}^{*}} \frac{e^{n \Psi_{n}(t)}}{t-z} d t, \quad \gamma_{n}^{*}=\gamma_{n} \backslash B_{n}
$$


Taking into account (101),

$$
\left|I_{n}^{*}(z)\right| \leq \frac{e^{n \Psi_{n}\left(t_{+}\right)}}{\operatorname{dist}\left(z, \gamma_{n}^{*}\right)} \exp \left(-\frac{\delta^{2}}{2 a^{3 / 2}} n^{1 / 2}\right), \quad z \notin \gamma_{n}^{*} .
$$

Asymptotics for $F_{n}$ can be obtained using AS72, Formula 7.1.23],

$$
F_{n}(t)=\frac{e^{n \Psi_{n}\left(t_{+}\right)}}{2 \sqrt{\pi}} \frac{a^{3 / 4}}{n^{3 / 4}\left(t-t_{+}\right)}\left(1+\mathcal{O}\left(\frac{1}{n^{1 / 2}}\right)\right), \quad t \in \beta_{n}
$$

also for $z \in B_{n} \backslash \gamma_{n}$,

$$
\left|\frac{1}{2 \pi i} \int_{\beta_{n}} \frac{F_{n}(t)}{t-z} d t\right| \leq \frac{C n^{-1 / 4} e^{n \Psi_{n}\left(t_{+}\right)}}{\operatorname{dist}\left(z, \beta_{n}\right)} .
$$

These estimates show that, roughly speaking, in the expressions (103) for $I_{n}$ function $F_{n}$ dominates in $B_{n}$, while its Cauchy transform along $\beta_{n}$ does it outside, and in consequence,

$$
I_{n}(z)= \begin{cases}-\frac{t_{+}^{n} \mathcal{F}\left(t_{+}\right)}{t_{+}-z} \frac{1}{2 \sqrt{\pi}}\left(\frac{a}{n}\right)^{3 / 4}\left(1+\mathcal{O}\left(\frac{1}{n^{1 / 2}}\right)\right)+\varepsilon_{1}(z), & \text { if } z \in \mathbb{C} \backslash\left(\overline{B_{n}} \cup \gamma_{n}^{*}\right), \\ F_{n}(z)+\varepsilon_{2}(z), & \text { if } z \in B_{n} \backslash \gamma_{n},\end{cases}
$$

where

$$
\left|\varepsilon_{1}(z)\right| \leq \frac{t_{+}^{n} \mathcal{F}\left(t_{+}\right)}{\operatorname{dist}\left(z, \gamma_{n}^{*}\right)} \exp \left(-\frac{\delta^{2}}{2 a^{3 / 2}} n^{1 / 2}\right), \quad\left|\varepsilon_{2}(z)\right| \leq \frac{C n^{-1 / 4} t_{+}^{n} \mathcal{F}\left(t_{+}\right)}{\operatorname{dist}\left(z, C_{n}\right)} .
$$

This asymptotic expression has several corollaries. First, formulas (98) show that $f_{n}^{(1)}$ should have no zeros inside $\gamma_{n}$. All zeros lie outside $\gamma_{n}$ and approach the circle $\mathbb{T}_{a}$ with the clock pattern following asymptotically the solutions of the equation

$$
\left(\frac{z}{t_{+}}\right)^{n}=\frac{1}{2 \sqrt{\pi}}\left(\frac{a}{n}\right)^{3 / 4} \frac{1}{z-t_{+}} \frac{\mathcal{F}\left(t_{+}\right)}{\mathcal{F}(z)}
$$

Away from $t_{+}$they should be close to the level curve $\operatorname{Re}\left(\Psi_{n}(z)-\Psi_{n}\left(t_{+}\right)\right)=\frac{1}{n} \log \left(\frac{1}{2 \sqrt{\pi}} \frac{a^{3 / 4}}{n^{3 / 4}}\right)$ (compare with Fig. 10, left), although at the boundary $\beta_{n}$ of the disk $B_{n}$ they approach the level curve $\operatorname{Re}\left(\Psi_{n}(z)-\Psi_{n}\left(t_{+}\right)\right)=\frac{1}{n} \log \left(\frac{1}{2 \sqrt{\pi}} \frac{a^{3 / 4}}{n^{1 / 4}}\right)$ (see Fig. [10] right).

Furthermore, the Verblunsky coefficients $\alpha_{n}$ for $\Phi_{n}$ exhibit the following asymptotic behavior:

$$
\alpha_{n}=-\frac{1}{2 \sqrt{\pi}} t_{+}^{n} \mathcal{F}\left(t_{+}\right)\left(\frac{a}{n}\right)^{3 / 4}\left(1+\mathcal{O}\left(\frac{1}{n^{1 / 2}}\right)\right), \quad n \rightarrow \infty .
$$

A new feature is the fractional power of $n$ in (105), not appearing when the singularities of $\mathcal{F}$ on $\mathbb{T}_{\rho}$ are only poles (cf. Corollary 3 ). 

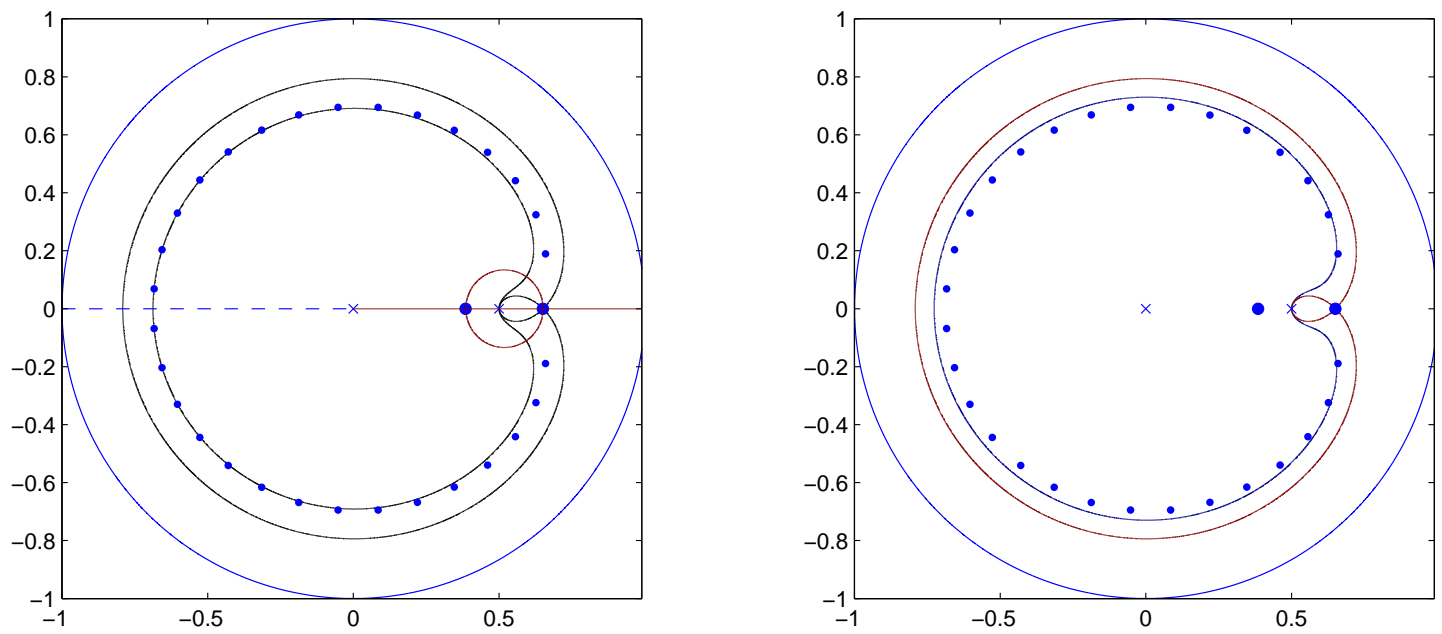

Figure 10: Zeros of $\Phi_{n}$ for $w$ given in (95) with $a=1 / 2$ and $n=30$, along with the level curves $\operatorname{Re}\left(\Psi_{n}(z)-\Psi_{n}\left(t_{+}\right)\right)=0, \operatorname{Re}\left(\Psi_{n}(z)-\Psi_{n}\left(t_{+}\right)\right)=\frac{1}{n} \log \left(\frac{1}{2 \sqrt{\pi}} \frac{a^{3 / 4}}{n^{3 / 4}}\right)$ and $\operatorname{Im}\left(\Psi_{n}(z)-\Psi_{n}\left(t_{+}\right)\right)=0$ (left). Picture on the right shows that zeros of $\Phi_{30}$ in a neighborhood of the singular point $a$ follow more closely the level curve $\operatorname{Re}\left(\Psi_{n}(z)-\Psi_{n}\left(t_{+}\right)\right)=\frac{1}{n} \log \left(\frac{1}{2 \sqrt{\pi}} \frac{a^{3 / 4}}{n^{1 / 4}}\right)$, as predicted.

Consider now the inverse of the weight function given in (95) by setting

$$
w(t)=\left|\exp \left(\frac{1}{t-a}\right)\right|^{2}, \quad t \in \mathbb{T}_{1},
$$

with $0<a<1$. Surprisingly we observe now a rather different behavior of the zeros of $\left\{\Phi_{n}\right\}$ (cf. Fig. 13) which is explained by the following analysis. For $w$ in (106),

$$
\mathcal{F}(t)=\exp \left(\frac{t}{1-a t}+\frac{1}{a-t}\right)
$$

and

$$
\Psi_{n}(t) \stackrel{\text { def }}{=} \log t+\frac{1}{n} \log \mathcal{F}(z)=\log t+\frac{1}{n}\left(\frac{t}{1-a t}+\frac{1}{a-t}\right)
$$

has saddle points

$$
t_{ \pm} \rightarrow a, \quad 1 / t_{ \pm} \rightarrow 1 / a
$$

satisfying

$$
t_{+}=\overline{t_{-}}, \quad t_{ \pm}=a \pm i \frac{\sqrt{a}}{\sqrt{n}}+\mathcal{O}(1 / n), \quad n \rightarrow \infty
$$




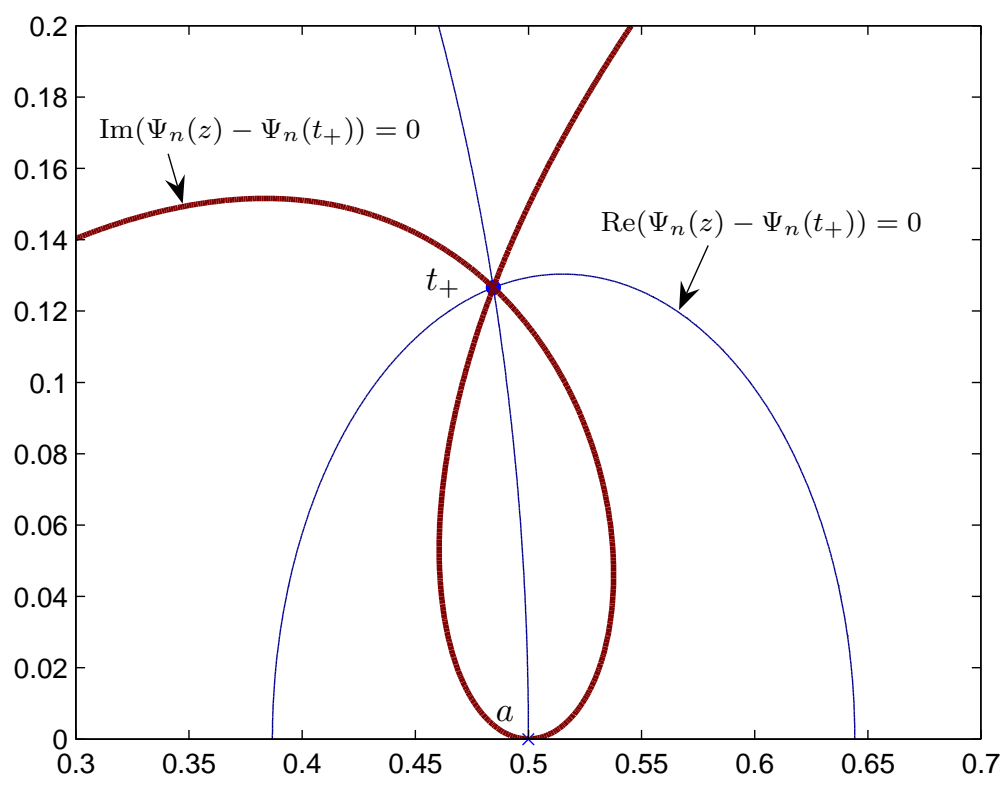

Figure 11: Level curves $\Gamma$ (thick lines) and $\Gamma^{\perp}$ in a neighborhood of $t_{+}$for $a=1 / 2$ and $n=30$.

Observe that in this case we have no dominant saddle point in a neighborhood of $a$.

An analysis as for weight (95) allows us to conclude that the local structure of the trajectories $\Gamma=\left\{t \in \mathbb{C} \backslash(-\infty, 0]: \operatorname{Im}\left(\Psi_{n}(t)-\Psi_{n}\left(t_{+}\right)\right)=0\right\}$ and $\Gamma^{\perp}=\{t \in \mathbb{C} \backslash(-\infty, 0]$ : $\left.\operatorname{Re}\left(\Psi_{n}(t)-\Psi_{n}\left(t_{+}\right)\right)=0\right\}$ is now as depicted in Figure 11] which yields in turn the global structure is as in Figure 12 Now we take as $\gamma_{n}$ in (97) the union of arcs of $\Gamma$ lying in the domain where $\operatorname{Re}\left(\Psi_{n}(z)-\Psi_{n}\left(t_{+}\right)\right)<0$ (see Fig. 12, right), except that we slightly depart from $\Gamma$ in an arbitrary small neighborhood to the right of $a$, where the inequality $\operatorname{Re}\left(\Psi_{n}(z)-\Psi_{n}\left(t_{+}\right)\right)<0$ is still valid.

Along with the notation introduced in (99), (100) and (102), we use and $B_{n}^{*}=\{\bar{z}: z \in$ $\left.B_{n}\right\}, \beta_{n}^{*}=\partial B_{n}^{*}$, and

$$
F_{n}(z) \stackrel{\text { def }}{=} P_{n}\left(\zeta_{n}(z)\right), z \in B_{n} \backslash \gamma_{n}, \quad F_{n}^{*}(z) \stackrel{\text { def }}{=} P_{n}\left(\overline{\zeta_{n}(\bar{z})}\right), z \in B_{n}^{*} \backslash \gamma_{n}
$$



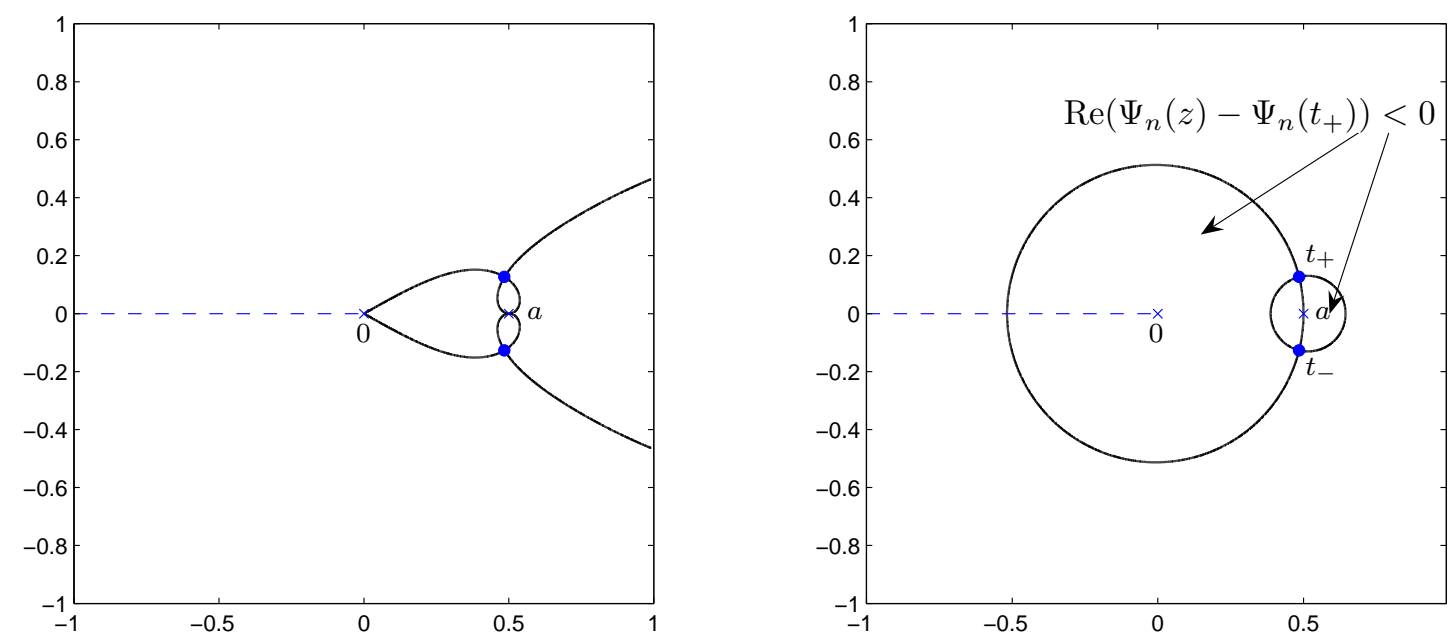

Figure 12: Global structure of the level curves $\Gamma$ (left) and $\Gamma^{\perp}$ for $a=1 / 2$ and $n=30$.

Following the arguments similar to those yielding (103) we obtain

$$
I_{n}(z)= \begin{cases}F_{n}(z)+I_{n}^{*}(z)-\frac{1}{2 \pi i} \int_{\beta_{n}} \frac{F_{n}(t)}{t-z} d t-\frac{1}{2 \pi i} \int_{\beta_{n}^{*}} \frac{F_{n}^{*}(t)}{t-z} d t, & \text { for } z \in B_{n} \backslash \gamma_{n}, \\ F_{n}^{*}(z)+I_{n}^{*}(z)-\frac{1}{2 \pi i} \int_{\beta_{n}} \frac{F_{n}(t)}{t-z} d t-\frac{1}{2 \pi i} \int_{\beta_{n}^{*}} \frac{F_{n}^{*}(t)}{t-z} d t, & \text { for } z \in B_{n}^{*} \backslash \gamma_{n}, \\ I_{n}^{*}(z)-\frac{1}{2 \pi i} \int_{\beta_{n}} \frac{F_{n}(t)}{t-z} d t-\frac{1}{2 \pi i} \int_{\beta_{n}^{*}} \frac{F_{n}^{*}(t)}{t-z} d t, & \text { for } z \in \mathbb{C} \backslash\left(\overline{B_{n}} \cup B_{n}^{*} \cup \gamma_{n}^{*}\right),\end{cases}
$$

where $I_{n}^{*}$ is as in (104) with $\gamma_{n}^{*}=\gamma_{n} \backslash\left(B_{n} \cup B_{n}^{*}\right)$.

With contour $\gamma_{n}$ described above we can perform the asymptotic analysis of the right hand sides in (107); again $F_{n}$ and $F_{n}^{*}$ will dominate in $B_{n}$ and $B_{n}^{*}$, respectively, but the leading term outside these discs will be given by the sum of their Cauchy transforms. In particular,

$$
I_{n}(z) \sim-\frac{1}{2 \sqrt{\pi}}\left(\frac{a}{n}\right)^{3 / 4}\left(\frac{t_{+}^{n} \mathcal{F}\left(t_{+}\right)}{t_{+}-z}+\frac{t_{-}^{n} \mathcal{F}\left(t_{-}\right)}{t_{-}-z}\right), \quad z \in \mathbb{C} \backslash\left(\overline{B_{n}} \cup \overline{B_{n}^{*}} \cup \gamma_{n}^{*}\right),
$$

and $I_{n}(z)$ has (asymptotically) at most one zero inside $\gamma_{n}$, originated by the cancelation of these Cauchy transforms. By (98), the rest of the zeros of $f_{n}^{(1)}$ lies outside $\gamma_{n}$ and distributes asymptotically as the solutions of the equation

$$
z^{n} \mathcal{F}(z)=\frac{1}{2 \sqrt{\pi}}\left(\frac{a}{n}\right)^{3 / 4}\left(\frac{t_{+}^{n} \mathcal{F}\left(t_{+}\right)}{t_{+}-z}+\frac{t_{-}^{n} \mathcal{F}\left(t_{-}\right)}{t_{-}-z}\right) .
$$



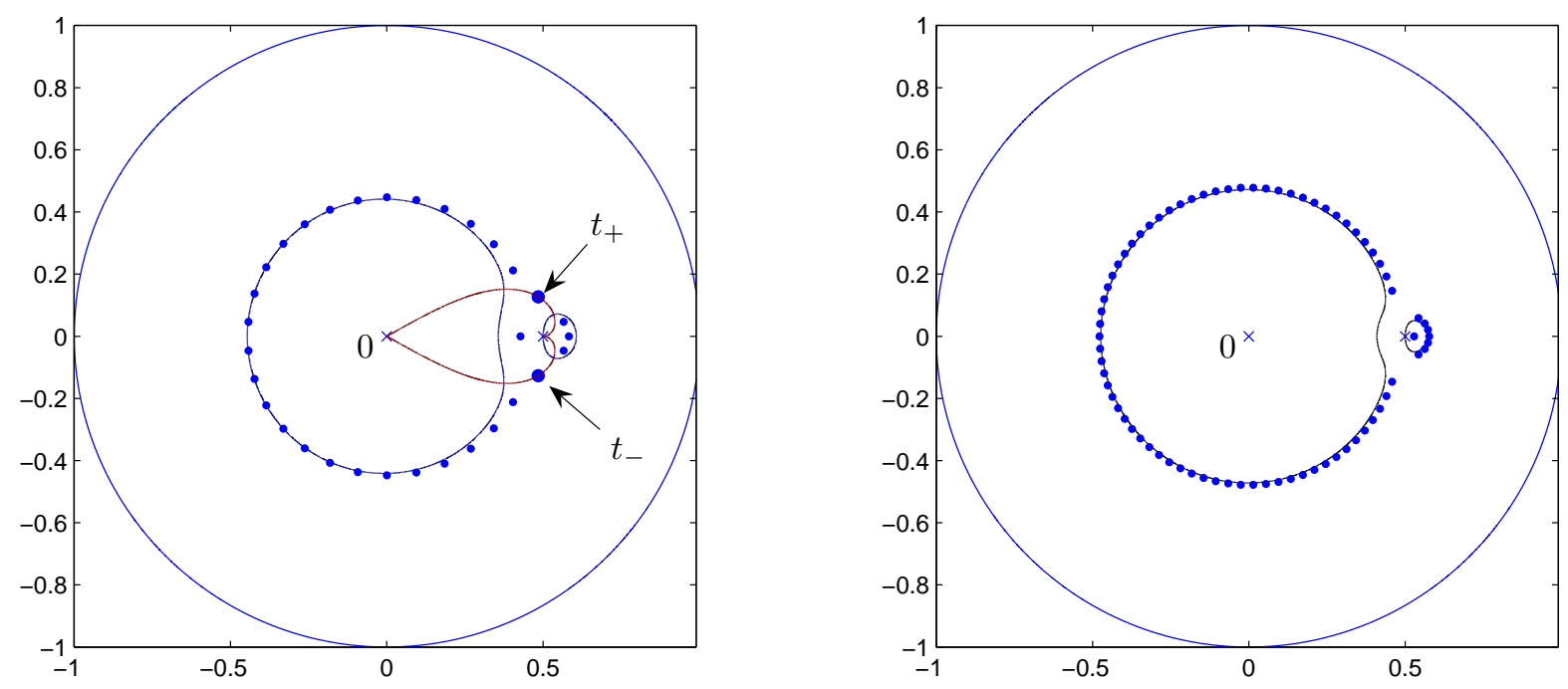

Figure 13: Left: zeros of $\Phi_{n}$ for $w$ given in (106) with $a=1 / 2$ and $n=30$, contour $\gamma_{n}$, and the level curve $\operatorname{Re}\left(\Psi_{n}(z)-\Psi_{n}\left(t_{+}\right)\right)=\frac{1}{n} \log \left(\frac{1}{2 \sqrt{\pi}} \frac{a^{3 / 4}}{n^{3 / 4}}\right)$ (which has two components). Right: same, but with $n=75$.

Hence, away from $\gamma_{n}$ they should be close again to the level curve $\operatorname{Re}\left(\Psi_{n}(z)-\Psi_{n}\left(t_{+}\right)\right)=$ $\frac{1}{n} \log \left(\frac{1}{2 \sqrt{\pi}} \frac{a^{3 / 4}}{n^{3 / 4}}\right)$ (compare with Fig. 13).

Finally, the Verblunsky coefficients $\alpha_{n}$ of $\Phi_{n}$ satisfy

$$
\alpha_{n}=-\frac{1}{\sqrt{\pi}}\left(\frac{a}{n}\right)^{3 / 4} \operatorname{Re}\left(t_{+}^{n} \mathcal{F}\left(t_{+}\right)\right)\left(1+\mathcal{O}\left(\frac{1}{n^{1 / 2}}\right)\right), \quad n \rightarrow \infty .
$$

Remark 17 Although we have considered here the case that $D_{\text {e }}$ possesses a single isolated singularity, it is clear that the same analysis carries through under the more general assumption that $D_{\mathrm{e}}$ can be extended to a domain of the form $\left\{|z|>\rho^{\prime}\right\}$ with $\rho^{\prime}<\rho$ such that $D_{\mathrm{e}}$ possesses exactly one isolated essential singularity of the form (96) in this domain.

Remark 18 Extensions in various directions of the considerations in this section are imminently possible; what is really required is $(i)$ a domain $\left\{|z|>\rho^{\prime}\right\}$ on which it is known that $D_{\mathrm{e}}$ possesses a finite number of singularities and $(i i)$ a description of the behavior of $D_{\mathrm{e}}$ in a vicinity of each singularity. With this information, a complete asymptotic description of the function $f_{n}^{(1)}$ is within the reach of careful, and often creative, asymptotic analysis of integrals. 


\section{Acknowledgement}

The research of A.M.F. was supported, in part, by a research grant from the Ministry of Science and Technology (MCYT) of Spain, project code BFM2001-3878-C02, by Junta de Andalucía, Grupo de Investigación FQM229, and by Research Network "Network on Constructive Complex Approximation (NeCCA)", INTAS 03-51-6637. A.M.F. acknowledges also the support of the Ministry of Education, Culture and Sports of Spain through the travel grant PR2003-0104, and the hospitality of the Department of Mathematics of the Vanderbilt University, where this work was started.

The research of K.T.-R.M. was supported, in part, by the National Science Foundation under grants DMS-0451495 and DMS-0200749.

The research of E.B.S. was supported, in part, by the U.S. National Science Foundation under grant DMS-0296026.

Both A.M.F. and K.T.-R.M. acknowledge also a partial support of NATO Collaborative Linkage Grant "Orthogonal Polynomials: Theory, Applications and Generalizations", ref. PST.CLG.979738.

We are grateful to Profs. P. Deift, J. S. Geronimo and P. D. Miller for very interesting discussions, as well as to Prof. B. Simon for his tireless activity in this field that stimulated our research as well.

\section{References}

[AS72] M. Abramowitz and I. A. Stegun. Handbook of Mathematical Functions. Dover Publ., New York, 1972.

[AV88] M. P. Alfaro and L. Vigil. Solution of a problem of P. Turán on zeros of orthogonal polynomials on the unit circle. J. Approx. Theory, 53:195-197, 1988.

[BDJ99] J. Baik, P. Deift, and K. Johansson. On the distribution of the length of the longest increasing subsequence of random permutations. J. Amer. Math. Soc., 12(4):1119-1178, 1999.

[BRLLS01] D. Barrios Rolanía, G. López Lagomasino, and E. B. Saff. Asymptotics of orthogonal polynomials inside the unit circle and Szegö-Padé approximants. In Proceedings of the Fifth International Symposium on Orthogonal Polynomials, Special Functions and their Applications (Patras, 1999), volume 133, pages 171$181,2001$.

[CMV03] M. J. Cantero, L. Moral, and L. Velázquez. Five-diagonal matrices and zeros of orthogonal polynomials on the unit circle. Linear Algebra Appl., 362:29-56, 2003. 
[Cass57] J. W. S. Cassels. An introduction to Diophantine approximation. Cambridge Tracts in Mathematics and Mathematical Physics, No. 45. Cambridge University Press, New York, 1957.

[Dei99a] P. A. Deift. Orthogonal polynomials and random matrices: a Riemann-Hilbert approach. New York University Courant Institute of Mathematical Sciences, New York, 1999.

[Dei99b] P. A. Deift. Integrable operators. In Differential operators and spectral theory, 69-84, Amer. Math. Soc. Transl. Ser. 2, 189, Amer. Math. Soc., Providence, RI, 1999.

[FIK92] A. S. Fokas, A. R. Its, and A. V. Kitaev. The isomonodromy approach to matrix models in 2D quantum gravity. Comm. Math. Phys., 147:395-430, 1992.

[GC79] J. S. Geronimo and K. M. Case. Scattering theory and polynomials orthogonal on the unit circle. J. Math. Phys., 20(2):299-310, 1979.

[Ger61] Ya. L. Geronimus. Orthogonal Polynomials: Estimates, Asymptotic Formulas, and Series of Polynomials Orthogonal on the Unit Circle and on an Interval. Consultants Bureau, New York, 1961.

[GM91] E. Godoy and F. Marcellán. An analogue of the Christoffel formula for polynomial modification of a measure on the unit circle. Boll. Un. Mat. Ital. A, 5:1-12, 1991.

[GS84] U. Grenander and G. Szegő. Toeplitz forms and their applications. Chelsea Publishing Co., New York, second edition, 1984.

[IR92] M. E. H. Ismail and R. W. Ruedemann. Relation between polynomials orthogonal on the unit circle with respect to different weights. J. Approx. Theory, 71(1):3960, 1992.

[LS87] D. S. Lubinsky and E. B. Saff. Convergence of Padé Approximants of Partial Theta Functions and the Rogers-Szegö Polynomials. Constr. Approx., 3:331-361, 1987.

[MGH90] D. S. Mazel, J. S. Geronimo, and M. H. Hayes III. On the geometric sequences of reflection coefficients. IEEE Transactions on Acoustics, Speech, and Signal Processing, 38(10):1810-1812, October 1990.

[MM04] K. T.-R. McLaughlin and P. D. Miller. The $\bar{\partial}$ steepest descent method and the asymptotic behavior of polynomials orthogonal on the unit circle with fixed and exponentially varying nonanalytic weights. Preprint arXiv:math.CA/0406484, 2004 . 
[MS90] H. N. Mhaskar and E. B. Saff. On the distribution of zeros of polynomials orthogonal on the unit circle. J. Approx. Theory, 63:30-38, 1990.

[NT89] P. Nevai and V. Totik. Orthogonal polynomials and their zeros. Acta Sci. Math. (Szeged), 53(1-2):99-104, 1989.

[Pak87] L. Pakula. Asymptotic zero distribution of orthogonal polynomials in sinusoidal frequency estimation. IEEE Transactions on Information Theory, 33(4):569-576, July 1987.

[Sim04a] B. Simon. Orthogonal Polynomials on the Unit Circle I and II. AMS Colloquium Series. American Mathematical Society, Providence, RI, 2005.

[Sim04b] B. Simon. Fine structure of the zeros of orthogonal polynomials, I. A tale of two pictures. preprint, 2004.

[Sim04c] B. Simon. Fine structure of the zeros of orthogonal polynomials, II. OPUC with competing exponential decay. preprint, 2004.

[ST04] B. Simon and V. Totik. Limits of zeros of orthogonal polynomials on the circle. preprint, 2004.

[Ste70] E. M. Stein. Singular Integrals and Differentiability Properties of Functions, volume 30 of Princenton Mathematical Series. Princenton University Press, Princenton, 1970.

[Sza79] J. Szabados. On some problems connected with polynomials orthogonal on the complex unit circle. Acta Math. Acad. Scient. Hung., 33(1-2):197-210, 1979.

[Sze75] G. Szegö. Orthogonal Polynomials, volume 23 of Amer. Math. Soc. Colloq. Publ. Amer. Math. Soc., Providence, RI, fourth edition, 1975. 